\title{
Der Vermögensschutz von Gesellschaften gegenüber externer Einflussnahme - geprüft am Beispiel der $\mathrm{GmbH}$
}

\author{
Dr. Jens Prütting, Hamburg*
}

Inhaltsübersicht

ZGR 2015, 849-886

I. Einführung . . . . . . . . . . . . . . . . . . . . . . . 850

II. Anerkannte Haftungsmechanismen und Konkurrenzerwägungen . . . . . . . . . 850

1. Vertragliche Auskunftsbeziehung . . . . . . . . . . . . . . . . . 850

2. Vertragsnähe und rechtsgeschäftliches Vertrauen . . . . . . . . . . . . . 851

3. Der Tatbestand der Geschäftsanmaßung als Allzweckwaffe? . . . . . . . . 852

4. Deliktischer Schutz absoluter Rechte und Rechtsgüter . . . . . . . . . . 853

5. Haftung wegen Verletzung eines Schutzgesetzes . . . . . . . . . . . 857

6. \826 BGB - „Der schwarze Ritter“ . . . . . . . . . . . . . . . 862

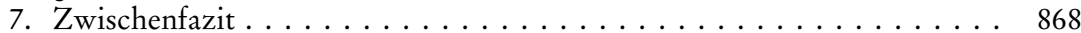

III. Zur analogen Anwendbarkeit des $₫ 117$ Abs. 1 AktG . . . . . . . . . . . . 868

1. Haftung nach $\$ 117$ Abs. 1 AktG . . . . . . . . . . . . . . . . 869

2. Gründe gegen eine Analogie zu $₫ 117$ Abs. 1 AktG . . . . . . . . . . . 875

3. Positivkriterien der Analogie . . . . . . . . . . . . . . . . . . 880

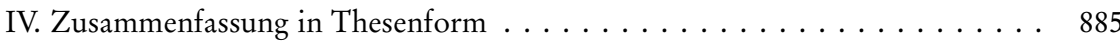

Das schlanke Recht der GmbH bietet kleinen und mittelständischen Unternehmen Bewegungsfreibeit, weist jedoch zugleich Lücken im System auf, die insbesondere mit dem umfangreichen Recht der AG geschlossen werden. Eine solche Regelungslücke existiert im Haftungsregime der GmbH zur Abwebr externer Einflussnabme unter Ausnutzung rechtsgeschäftlicher oder tatsächlicher Druckpositionen auf die Leitungs- und Führungsebene. Das Recht der AG löst diese Problemlage über $\int 117$ Abs. 1 AktG dabingehend, dass Schädigungen der Gesellschaft, die auf eine vorsätzliche Ausnutzung einer Druckposition eines Einflussnehmers zurückgefübrt werden können, zur Haftung gegenüber der Gesellschaft selbst fübren. Diese im Aktienrecht verankerte Strategie ist vor dem Hintergrund ibrer Geschichte, ibrer Systematik und ibrer Teleologie als allgemeines Deliktsrecht der Gesellschaftsformen aufzufassen und auf die $\mathrm{GmbH}$ analog anzuwenden.

* Prof. Dr. Jens PrütTing, LL.M. oec. (Köln), ist Juniorprofessor für das Recht der Familienunternehmen an der Bucerius Law School (Hamburg) und Habilitant am Institut für ausländisches und internationales Privat- und Wirtschaftsrecht der Universität Heidelberg (Prof. Marc-Philippe Weller). Der Aufsatz geht auf ein Lehrstuhlkolloquium der Institute Prof. Dr. Barbara Dauner-Lieb (Köln) und Prof. Dr. Marc-Philippe Weller (Heidelberg) zurück. Besonderer Dank gilt Charlotte Harms, Dr. Chris Thomale und Prof. Dr. Weller für die kritische Durchsicht und wertvolle Hinweise. 
The slim codex on German Limited Companies (GmbH) offers flexibility for small and medium-sized businesses, however leaves regulatory gaps which are often closed by applying the more comprehensive laws on Stock Corporations. Within the liability regime of Limited Companies, such a gap exists concerning external influence on the executive and management level by unduly taking advantage of circumstances that constitute a moment of contractual or factual pressure. In the laws on Stock Corporations, a person is made liable for damage caused to the corporation by willful influence of this kind. This regulatory strategy for Stock Corporations, when seen in historic, systematic and teleological light, constitutes a general rule of tort for all corporations and is thus to be applied to Limited Companies (GmbH) as well.

\section{Einfübrung}

Die vorliegende Untersuchung setzt sich mit der Haftung externer Einflussnehmer auf eine $\mathrm{GmbH}$ auseinander, die ihrerseits zwar gesellschaftsrechtlich nicht oder nicht alleine über Verwaltungsrechte verfügen, die jedoch auf Basis faktischer Positionen Einflussnahmemöglickeiten besitzen. Zu denken ist an Ehegatten von Verwaltungspersonen der Gesellschaft, die auf Basis heimischer Einflussnahme eine Entscheidung des Geschäftsführers bewirken, durch welcher der Gesellschaft ein Schaden entsteht. Dabei erkennt der einflussnehmende Partner das Risiko und nimmt den Schaden billigend in Kauf. Seine Ambitionen werden häufig auf Gewinne für die Gesellschaft gerichtet sein und dem Wunsch nach Mitspracherechten entspringen. Demgegenüber mag der geschäftsführende Ehegatte das Risiko erkannt und gleichwohl gehandelt oder aber der Gesellschaft irrtümlich geschadet haben. Einen stärkeren Bezug zur geschädigten Gesellschaft weisen daneben jene Fallkonstellationen auf, in denen der Einflussnehmer etwa zugleich als Lieferant oder wichtiger externer Vertragspartner, aber auch als beteiligter Gesellschafter seine Machtstellung in Bezug auf die $\mathrm{GmbH}$ nutzt, um deren Geschicke über die Lenkung eines Organs der Leitungs- und Führungsebene zu steuern.

Im Folgenden werden Haftungsansprüche der Gesellschaft in Form der $\mathrm{GmbH}$ gegen den Einflussnehmer an Hand des gegebenen bürgerlich-rechtlichen Haftungsinstrumentariums geprüft (II), um sodann in eine Diskussion über einen denkbaren Analogieschluss zu $\$ 117 \mathrm{Abs.} 1 \mathrm{AktG}$ einzutreten (III). Die Ergebnisse werden abschließend in Thesenform zusammengefasst (IV).

\section{Anerkannte Haftungsmechanismen und Konkurrenzerwägungen}

\section{Vertragliche Auskunftsbeziebung}

Sofern der Einflussnehmer mit der Gesellschaft in Bezug auf die schadensstiftende unternehmensleitende Handlung in vertraglicher Verbundenheit 


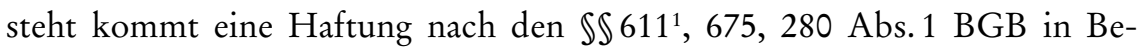
tracht ${ }^{2}$. Soweit ein Schaden kausal auf die fehlerhafte Beratung zurückgeführt werden kann, handelt es sich um einen Haftungsfall. Dabei genügt jeder Grad der Sorgfaltswidrigkeit, $\$ 276$ Abs. 1, 2 BGB. Für die Verjährung gelten die $\mathbb{S} 195,199$ Abs. 1 BGB, für die Haftungszurechnung $\$ 278$ 2. Alt. BGB - oder je nach Einzelfall $\$ 31$ BGB analog ${ }^{3}$-, so dass dahinter eine deliktische Haftung nicht mehr von praktischem Interesse ist ${ }^{4}$. Dass das Vermögen im vertraglichen Gefüge einen derart weitreichenden Schutz genießt, ist einleuchtend, führt doch die von allen Vertragsparteien bewusst herbeigeführte Nähe zu einem erleichterten Zugriff auf die Rechte und Interessen des anderen Teils, wodurch in erhöhtem Maße die Gefahr von Schädigungen besteht und diesen Umstand ausgleichend die Sorgfaltsanforderungen durch entsprechende Haftungstatbestände allen Beteiligten verschärft in das Bewusstsein zu rücken sind ${ }^{5}$. Dies ist auch im Rahmen des Ausgleichs zu den geschäftlichen Chancen zu sehen, die die Vertragsparteien mit ihrem Verhalten erwerben ${ }^{6}$.

Der Ansatz deckt jedoch die vorliegende Problematik nicht ab, da die praktisch zu lösenden Konstellationen gerade Personen inkludieren, die Einfluss aufgrund nur faktischer Positionen nehmen (Emotionalitäten, keine fortlaufenden Belieferungen als bedeutender Zulieferer etc.). Gleichwohl ist der Ansatz im Haftungssystem bedeutsam, da die Rechtsprechung über die Zeit eine Tendenz zeigt, bereits aus besonderen Umständen, die jeweils beiden Parteien bekannt sind, und dem sodann erfolgenden Rat die konkludente Vereinbarung eines Auskunfts- oder Beratungsvertrages herzuleiten 7 . Der Haftungsgrund liegt insofern aber nicht in der Einflussnahme als solcher, wie diese durch ein klassisches Jedermann-Delikt unterbunden würde.

\section{Vertragsnähe und rechtsgeschäftliches Vertranen}

Da zwischen Einflussnehmer und diesem folgendem Gesellschaftsorgan regelmäßig kein vertragliches Verhältnis in Bezug auf die Einflussnahme ent-

1 Bei nur einmaliger, spezifischer Beratungsleistung als Werkvertrag einzustufen, vgl. BGH NJW 1999, 1540.

2 Vgl. Sprau, in: Palandt, BGB, 74. Aufl., 2015, $\$ 675$ Rdn. 35. $\$ 675$ Abs. 2 BGB ist rein deklaratorisch zu verstehen und bezieht sich ausschließlich auf Beratung und Auskunft im Rahmen von Gefälligkeitsverhältnissen, vgl. HeErmann, Münchener Komm. z. BGB, 6. Aufl., 2012, $\$ 675 \mathrm{Rdn} .112$ f m. w. N.

3 Allg. M. vgl. nur Ellenberger, in: Palandt, BGB, 74. Aufl., 2015, $\$ 31$ Rdn. 3 m. w. N.

4 Allenfalls für die Begründung des deliktischen Gerichtsstandes nach Art. 5 Nr.3 EuGVVO resp. 32 ZPO vs Art. 5 Nr. 1 EuGVVO resp. 29 Abs. 1 ZPO mag der Unterschied im Einzelfall noch interessieren.

5 Allg. M. vgl. statt vieler CANARIs, Schutzgesetz - Verkehrspflichten - Schutzpflichten, FS Larenz II, 1983, S. 30 ff, 88.

6 Vgl. Welser, Vertretung ohne Vollmacht, 1970, S. $76 \mathrm{f}$.

7 Vgl. Wagner, Münchener Komm. z. BGB, 6. Aufl., 2013, $\$ 826$ Rdn. 67 f m. w. N. 
stehen wird - Ausnahme bleibt die oben angeführte Auskunftssituation -, kann die Gesellschaft weder über das Konstrukt des Vertrages mit Schutzwirkung zu Gunsten Dritter ${ }^{8}$ noch über das Vehikel des $\$ 311$ Abs. 3 BGB ${ }^{9}$ einbezogen werden ${ }^{10}$. Eine Haftungsausweitung in diesem Bereich kommt nicht in Betracht ${ }^{11}$. Die quasivertraglichen Haftungsinstrumente basieren auf der Überlegung, dass zwischen anderen Personen ein Rechtsverhältnis besteht, welches wenigstens rechtsgeschäftliche Strukturen aufweist ${ }^{12}$ und somit auch ähnlichen Argumentationsmustern einer verschärften Haftung folgt ${ }^{13}$.

\section{Der Tatbestand der Geschäftsanmaßung als Allzweckwaffe?}

Wird die Einflussnahme als geschäftliche Handlung i. S. d. $\$ \$ 677 \mathrm{ff}$ BGB aufgefasst $^{14}$, ist eine Haftung des Einflussnehmers nach den $\int \ 687$ Abs. 2 Satz 1, 678 sowie 677, 280 Abs. 1 BGB wegen Übernahme ${ }^{15}$ und Ausführungsverschuldens ${ }^{16} \mathrm{zu}$ erwägen. Folgte man diesem Ansatz, ergäbe sich hieraus eine umfassende Haftung eines jeden Einflussnehmers, der die Fremdheit der manipulierten Interessenlagen erkennt und sich deren Gegenstand als eigenes Geschäft anmaßt ${ }^{17}$.

8 Zur Entwicklung, Rechtsnatur und Voraussetzungen GotTwald, Münchener Komm. z. BGB, 6. Aufl., 2012, $\$ 328$ Rdn. 161 m. w. N.

9 Zu den Voraussetzungen Emmerich, Münchener Komm. z. BGB, 6. Aufl., 2012, $\$ 311$ Rdn. $185 \mathrm{ff}$.

10 Es bedarf sowohl beim Vertrag mit Schutzwirkung für Dritte als auch im Rahmen des \311 Abs. 3 BGB einer anknüpfungsfähigen, rechtsgeschäftlichen Handlung, vgl. die Rechtsprechungsanknüpfung BGH NJW 1977, 2073, 2076. S. a. BydLINsKi, JBl. 1960, 359, $361 \mathrm{ff}$ mit der Forderung des Bestehens eines Vertrages. Ebenso Gernhuber, FS Nikisch, 1958, S. 249, 268. Demgegenüber lassen BERG, MDR 1969, 613, 615 und CANARIs, ZHR 163 (1999), 206 ein quasivertragliches Schuldverhältnis in Form einer c.i.c. ausreichen. Vgl. zur Diskussion auch Junker, Die Vertretung im Vertrauen im Schadensrecht, 1991, S. 68.

11 Gegenteiliges wäre mit der anerkannten Rechtsgrundlage nicht mehr vereinbar und wird auch nicht vertreten, vgl. GotTwald, aaO (Fn. 8), $\$ 328$ BGB Rdn. 165 f m. w. N.

12 GotTwald, aaO (Fn. 8), \$328 BGB Rdn. 165 f m. w. N.

13 Grundlegend Wilburg, Die Entwicklung eines beweglichen Systems im bürgerlichen Recht, 1950, S. 162 ff; vgl. a. KozIol, ZEuP 1995, 359, 364 f m. w. N.

14 Die geschäftliche Handlung dürfte kaum einmal zu verneinen sein, vgl. BGHZ 38, $270=$ NJW 1963, 390; Bergmann, in: Staudinger, BGB, 2015, Vor $\$ \int 677 \mathrm{ff} R d n .7$ f; Dornis, in: Erman, BGB, 14. Aufl., 2014, $\$ 677$ Rdn. 2.

15 Vgl. Sprau, aaO (Fn. 2), \677 BGB Rdn. 13; Grüneberg, in: Palandt, BGB, 74. Aufl., 2015, $\$ 280$ Rdn. 9.

16 Vgl. SeIler, Münchener Komm. z. BGB, 6. Aufl., 2012, \678 Rdn. 1.

17 Die Voraussetzungen ergeben sich im Einzelnen aus der gesetzlichen Bestimmung. Vgl. Bergmann, aaO (Fn. 14), $\$ 687$ BGB Rdn. $1 \mathrm{ff}$. 
Allerdings müsste es sich bei der Beeinflussung eines Dritten zur Manipulation der Handhabung bestehender Pflichten dieser Person um ein objektiv fremdes Geschäft i.S.d. $\$ 677 \mathrm{ff}$ BGB handeln ${ }^{18}$. Die Einflussnahme müsste dem Rechts- und Interessenkreis des von der folgenden Verhaltensänderung Betroffenen zuzurechnen $\operatorname{sein}^{19}$. Dies ist abzulehnen. Streitig ist bereits, ob eine Geschäftsanmaßung in Form der eigenständigen angemaßten Geschäftsführung eines Unternehmens den Tatbestand des $\$ 687$ Abs. 2 BGB auszulösen geeignet ist, da auch die Geschäftsanmaßung in Parallellauf zu $\ 823$ Abs. 1 BGB den Schutz absoluter Rechte avisieren soll ${ }^{20}$. Daneben darf konkurrenzrechtlich die Haftung aus Geschäftsanmaßung nicht isoliert betrachtet werden, sondern ist in den haftungsrechtlichen Kontext des Deliktsrechts einzustellen ${ }^{21}$. Die Haftung aus $₫ 687$ Abs. 2 Satz 1 BGB bildet eine Einstandspflicht für den vorsätzlichen, rechtswidrigen Übergriff in fremde Rechts- und Interessenkreise. Diese müssen bestimmbar sein und können nicht mit einem allgemeinen Hinweis auf mittelbare Vermögensabflüsse ersetzt werden ${ }^{22}$.

\section{Deliktischer Schutz absoluter Rechte und Rechtsgüter}

Schutz des primären Vermögens wird über die zentrale Haftungsnorm des allgemeinen Deliktsrechts in $\$ 823$ Abs. 1 BGB nicht vermittelt ${ }^{23}$. Reinen Vermögensschutz sollen die $\int \mathbb{S} 823$ Abs. 2 und 826 BGB unter ihren besonderen Voraussetzungen bieten ${ }^{24}$. Dies ist als Grundsatzerwägung des Gesetzes zu

18 Der Begriff des objektiv fremden Geschäfts ist innerhalb der $\$ \$ 677-87$ BGB einheitlich, vgl. SEILER, aaO (Fn. 16), $\$ 687$ BGB Rdn. 8.

19 Ausführlich mit zutreffender Kritik an der Unbestimmtheit aller Definitionsversuche Brennecke, Ärztliche Geschäftsführung ohne Auftrag, 2010, S. 3 ff; s. a. SEILER, aaO (Fn. 16), \$677 BGB Rdn. 4 ff. Zu Hintergrund und Entstehungsgeschichte vgl. REICHARD, AcP 193 (1993), 567 ff.

20 Ablehnend BGHZ 7, 208, 218 = NJW 1952, 1410; Bergmann, in: Staudinger, 2015, \687 Rdn. 24; DäUbler, JuS 1969, 49, 53. Bejahend dagegen SeIler, aaO (Fn. 16), \687 BGB Rdn. 20, wenngleich zutreffend darauf hingewiesen wird, dass $\$ 687$ BGB den Schutz absoluter Rechtsgüter als Parallele zu $\ 823$ Abs. 1 BGB vor Augen haben sollte, vgl. SeIler, aaO, Rdn. 19 m. w. N.

21 Vgl. SeILer, aaO (Fn. 16), \687 BGB Rdn. 19.

22 Vor diesem Hintergrund greift auch die Rspr. in den $\$ \mathbb{S} 677 \mathrm{ff}$ BGB auf das Kriterium der Unmittelbarkeit zurück, vgl. BGHZ 55, 207 = NJW 1971, 754. Die Rspr. verweist auf das Recht am Unternehmen als hier einzig denkbare Position und lehnt einen hinreichend abgrenzbaren Schutzumfang ab, vgl. BGHZ 43, 359, 361; Bergmann, aaO (Fn. 20), $\$ 687$ BGB Rdn. 24 m.V.a. BGHZ 71, 86, 98.

23 So schon RGZ 51, $92 \mathrm{f}$ und seither st. Rspr.; vgl. in späterer Zeit BGHZ 27, 137, $140=$ NJW 1958, 1041 f; BGHZ 86, 152, 155 = NJW 1983, 2313 f; HAGER, in: Staudinger, 1999, \823 Rdn. B 192 m. w. N.; S. a. Jansen, Struktur des Haftungsrechts, 2003, S. 524 ff.

24 Einander gegenüberstellend WaGNER, aaO (Fn. 7), $\ 823$ BGB Rdn. 207, 247 ff m. w. N. 
begrüßen, will doch eine liberale Marktordnung im Zweifel den Leistungswettbewerb den freien Kräften überlassen und eben erst dort mittels Haftungsrecht ressourcenallozierend eingreifen, wo Marktversagen erkannt wird $^{25}$.

Die Einflussnahme in den hier interessierenden Konstellationen führt jedoch regelmäßig weder zu einer Verletzung der aufgezählten noch der sonstigen geschützten Rechtsgüter. Rechtsgüterschutz verlangt in $\$ 823$ Abs. 1 BGB nämlich dem Grunde nach Ausschließlichkeit, Offenkundigkeit und einen hinreichend bestimmbaren Zuweisungsgehalt der verteidigten Position $^{26}$.

Einen interessanten Diskussionsansatz bietet allenfalls das Recht am eingerichteten und ausgeübten Gewerbebetrieb ${ }^{27}$, auch Recht am Unternehmen ${ }^{28}$ genannt. Dieses Rechtsinstitut soll dann eingreifen, wenn ein vermeidbarer betriebsbezogener Übergriff nach einer umfassenden Interessen- und Güterabwägung sich als rechtswidrige Beeinträchtigung des Unternehmensbestandes darstellt und keine spezielle Regelung für den jeweiligen Fall ersichtlich ist $^{29}$. Hierdurch wird die gebotene Parallele zu $\$ 826$ BGB hergestellt, um einen allgemeinen primären Vermögensschutz im Deliktsrecht im Gewande einer Fahrlässigkeitshaftung nicht zu billigen ${ }^{30}$. Der Betriebsbezug verlangt die Anlehnung an die Vorsatzkomponente und die gebotene Interessenabwägung bildet das Gegengewicht zur Sittenwidrigkeitsforderung des $\$ 826$ $\mathrm{BGB}^{31}$. Bei Lichte betrachtet ist der geschützte Besitzstand am Unternehmen jedoch ein offen zu Tage liegender Versuch, den Schutz des primären Ver-

25 Vgl. MüHLEnKamp, „Marktversagen“ als ökonomische Begründung für Interventionen der öffentlichen Hand, in: Hrbek/Nettesheim, Europäische Union und mitgliedschaftliche Daseinsvorsorge, 2002, S. 65 ff. Eine Übersicht bieten Schäfer/OTт, Lehrbuch der ökonomischen Analyse des Zivilrechts, 2012, S. $78 \mathrm{ff}$.

26 Vgl. Koziol, ZEuP 1995, 359 unter Bezugnahme auf Mertens, Münchener Komm. z. BGB, 2. Aufl., XXXX (Jahr), $\$ 823$ Rdn. $101 \mathrm{ff}$.

27 Vgl. schon RGZ 58, 24, 29 ff und später BGHZ 3, 270, 278 ff = NJW 1952, 660 f; BGHZ 8, 142, 144 = NJW 1953, 297; SCHILDT, Der deliktische Schutz des Rechts am Gewerbebetrieb, WM 1996, 2261; SACK, Das Recht am Gewerbetrieb, 2007, S. $100 \mathrm{ff}$ mit scharfer Kritik an diesem Rechtsinstitut vgl. S. 142 ff. Ein „dubioses Recht“ gar nennt es Koziol, ZEuP 1995, 359, 361 und verweist darauf, dass diese Ausweitung des Vermögensschutzes etwa in Österreich keinen Fuß fassen könne. Ebenfalls kritisch K. SсHмIDT, JuS 1993, 985, 988.

28 So schon Fikentscher, FS Kronstein, 1967, S. 261 ff. Eingehende Analyse der Grenzen des Deliktsschutzes bei Habersack, Die Mitgliedschaft, 1996, S. $139 \mathrm{ff}$.

29 Hierzu Katzenmeier, in: Dauner-Lieb/Heidel/Ring, BGB, 2. Aufl., 2012, $₫ 823$ Rdn. $259 \mathrm{ff}$ m. w. N.

30 Vgl. WaGner, aaO (Fn. 7), $\$ 823$ BGB Rdn. 207, 256.

31 Hierzu BGH NJW 2003, 1040 f; Wagner, aaO (Fn. 7), $\$ 823$ BGB Rdn. 256. 
mögens partiell auch über $\int 823$ Abs. 1 BGB zu gewährleisten ${ }^{32}$. Mit Recht wurde daher darauf hingewiesen, dass $₫ 823$ Abs. 1 BGB als deliktische Generalklausel eine Entwicklungsfähigkeit zur Anpassung an veränderte Bedingungen innewohne ${ }^{33}$, der jedenfalls nur solche Rechte und Rechtsgüter zugänglich seien, die den oben genannten Kriterien genügen, die also insbesondere einen hinreichend bestimmbaren Zuweisungsgehalt besitzen ${ }^{34}$. Unabhängig davon vermag aber selbst in der heute durch die Rechtsprechung anerkannten Ausprägung besagter Ansatz in den Fällen rechtswidriger Beeinflussung nicht zu tragen, da es bereits an der Betriebsbezogenheit fehlt.

In partieller Abkehr von den Erfordernissen, die noch das RG aufgestellt hatte ${ }^{35}$, wird heute keine Bestandsgefährdung des Unternehmens mehr verlangt ${ }^{36}$. Allerdings muss sich der Eingriff unmittelbar, das heißt spezifisch betriebsbezogen gegen das Unternehmen richten ${ }^{37}$. Hierfür soll eine Beeinträchtigung der Entscheidungsfreiheit ausreichen können, wenn damit eine empfindliche Störung einhergeht ${ }^{38}$.

Ob diese Ansätze eine klare Linie ergeben können oder eher eine schlichte Ausklammerung diffuser Schadensbilder darstellen ${ }^{39}$, kann hier dahinstehen. Solange eine gesellschaftsexterne Person Einfluss auf ein Verwaltungsorgan nimmt, welches in der Folge aber gleichwohl eine selbstbestimmte Entscheidung noch zu treffen in der Lage ist ${ }^{40}$, kann Unmittelbarkeit des Eingriffs nicht angenommen werden. Es ist bereits fraglich, in welchem Moment der Eingriff in das Unternehmen stattfindet:

a) Stellt man auf die tatsächlich unternehmensnahe Entscheidung des Verwaltungsmitglieds nach bereits erfolgter Einflussnahme ab, ist es nicht der Externe, der das haftungsrelevante Verhalten zeigt, solange er den Handelnden nicht

32 Vgl. SAck, aaO (Fn. 27), S. 142 ff; Picker, ZfA 2010, 479, 573 ff.

33 Vgl. Deutsch, JZ 1963, 385, 388; Wagner, aaO (Fn. 7), \823 BGB Rdn. 205.

34 Vgl. Canaris, FS Steffen, 1995, S. 85, 90; Habersack, aaO (Fn. 28), 1996, S. 130 ff; Koziol, ZEuP 1995, $359 \mathrm{f}$.

35 RGZ 73, 107, 112; 77, 217 ff; 79, 224, 226; 102, 223, 225.

36 Anders schon RGZ 132, 311, 316; 158, 377, 379; 163, 21, 32 und in der Folge BGHZ 29, 65, 74 = NJW 1959, 479, 481; BGH NJW 2003, $1040 \mathrm{f}$.

37 BGHZ 29, 65, 74 = NJW 1959, 479, 481; s. a. HaGer, aaO (Fn. 23), \823 BGB Rdn. D 11.

38 BGHZ 138, 311, 317 f; BAG NJW 2009, 1990; s.a. WaGner, aaO (Fn. 7), \823 BGB Rdn. 257; KATZENMEier, aaO (Fn. 29), \823 BGB Rdn. 262.

39 Hierzu Wagner, aaO (Fn. 7), \823 BGB Rdn. 257, \826 Rdn. 17: Ausklammerung diffuser Schadensbilder als der mit „Abstand schwächste[...] Legitimationsgrund“.

40 Hinsichtlich mittelbarer Täterschaft wird im Deliktsrecht auf die Strafrechtsdogmatik, vgl. BGHZ 63, 124, 126 = NJW 1975, 49 f; BGHZ 164, 50, 57 = NJW 2005, 3137; BGHZ 184, 365 = VersR 2011, 750. Vgl. zur mittelbaren Täterschaft und zu den verschiedenen Formen der Annahme von Werkzeugqualität Fischer, StGB, 62. Aufl., 2015, $\mathbb{} 25$ Rdn. $4 \mathrm{ff} \mathrm{m.w.} \mathrm{N.}$ 
wie ein Werkzeug zu steuern vermag. Eine Gleichstellung über $\$ 830$ Abs. 1 Satz 1, Abs. 2 BGB $^{41}$ kommt hier gerade nicht in Betracht, da die Pflichtverletzung und folgende Innenhaftung des Verwaltungsmitglieds für den Externen nicht teilnahmefähig ist. Dies ergibt sich bereits aus der Rechtsnatur der $\mathbb{\int} 43$ Abs. 2 GmbHG, 93, 116 AktG, die rein gesellschaftsrechtlich - und damit vertragsnah - einzuordnen sind ${ }^{42}$ und somit einen Rückgriff auf das allgemeine Deliktsrecht aufgrund andersartiger Rechtsnatur nicht zulassen ${ }^{43}$. Die gegenteilige Sicht würde zu dem absurden Ergebnis führen, dass jeder Dritte im freien Leistungswettbewerb etwa bei für seine Mitbewerber unvorteilhafter Einflussnahme zu befürchten hätte, an der Haftung nach den $\$ \mathbb{S} 43$ Abs. 2 GmbHG, 93, 116 AktG über $\ 830$ BGB teilzunehmen. Dass dies nicht Telos der gesellschaftsrechtlichen Organhaftung sein kann, liegt auf der Hand.

b) Stellt man aber auf die Einflussnahme selbst als haftungsrelevantes Verhalten ab, so mag dieses Verhalten im Einzelfall sich sogar gegen das Unternehmen richten, jedoch ist es gerade dem betroffenen Verwaltungsmitglied nunmehr überlassen, seine Entscheidungen rein am Gesellschaftswohl auszurichten. Dieser unüberbrückbare Punkt in der Kausalkette bietet eine Kontrollinstanz, die gerade im täglichen Leistungswettbewerb dazu eingerichtet ist, für das Unternehmen sinnvolle von abzulehnenden Entscheidungen zu trennen ${ }^{44} . \mathrm{Ob}$, in welchem Rahmen und unter welchen Voraussetzungen gleichwohl eine Einflussnahme Dritter zur Haftung führen soll, ist damit der Einschätzungsprärogative einer gesetzgeberischen Entscheidung überlassen, die für das Aktienrecht in $\$ 117 \mathrm{Abs.} 1 \mathrm{AktG}$ auch genutzt wurde. Einer Erweiterung über deliktische Generalklauseln ist dieser Gedanke aber in dogmatischer und methodischer Hinsicht nicht zugänglich, da hiermit ein unkontrollierbarer Bruch mit dem Grundsatz des Verbots der Liquidation primärer Vermögensschäden über $\ 823$ Abs. 1 BGB einherginge und der Schutz sonstiger Rechte in $\$ 823$ Abs. 1 BGB noch konturloser würde, als dies jetzt bereits der Fall ist.

41 Es handelt sich um eine Zurechnungs- und nicht um eine bloße Beweislastregelung, vgl. BGHZ 8, 288, $292 \mathrm{ff}=$ NJW 1953, 499f; BGHZ 63, 124, 126 = NJW 1975, 49 f; BGHZ 137, 89, 102 = NJW 1998, 377, 381 f. Zum Streitstand s. WAGNER, aaO (Fn. 7), \$830 BGB Rdn. 4 f m.w. N.

42 Vgl. BGH NJW-RR 1989, 1255 f, weshalb vertragliche Ansprüche gegen den Geschäftsführer neben $\ 43 \mathrm{Abs} .2 \mathrm{GmbHG}$ sogar nach überwiegender Ansicht verdrängt werden, vgl. BGH NJW-RR 2008, 484. A.A. Scholz/Schneider, Komm. z. GmbHG, 11. Aufl., 2013, Band II, $\$ 43$ Rdn. 18.

43 Deliktsrecht kann nur in Anspruchskonkurrenz daneben zur Anwendung kommen, weist jedoch eine andere Rechtsnatur auf, da es gerade auf Einflüssen durch Jedermann basiert, vgl. BGH DB 2005, $821 \mathrm{f}$.

44 Entsprechende Überlegungen sind über die Schutznormlehre anzustellen, vgl. hierzu WAGNER, aaO (Fn. 7), \823 BGB Rdn. $366 \mathrm{ff}$. 


\section{Haftung wegen Verletzung eines Schutzgesetzes}

\823 Abs. 2 BGB bietet zwar den Schutz des primären Vermögens ${ }^{45}$, jedoch bedarf es hierfür der Verletzung eines Gesetzes, welches über den bloßen Schutz der Allgemeinheit hinaus ${ }^{46}$ sowohl personell als auch sachlich Deliktschutz mit Haftung des Schädigers auf Schadensersatz bezweckt ${ }^{47}$, und die geschützte Person mit ihrem geschützten Interesse gerade vor Schäden der im Einzelfall entstandenen Art bewahrt werden soll (Schutzzweckzusammenhang $)^{48}$. Es können verschiedene Vorschriften erwogen werden, die als Schutzgesetze im Bereich unzulässiger Einflussnahme anzusprechen sind:

\section{a) $\int 117 \mathrm{Abs} .1 \mathrm{AktG}$}

$\$ 117$ Abs. 1 AktG ist nach allgemeiner Meinung kein Schutzgesetz i.S.d. $\$ 823$ Abs. 2 BGB $^{49}$. Diesem Standpunkt ist zuzustimmen, bildet doch $\$ 117$ Abs. 1 AktG eine spezifische Anspruchsgrundlage, die gerade aus sich selbst heraus Schadensersatz gewährt und hierfür bestimmte Voraussetzungen aufstellt. Zudem ist $\$ 117$ Abs. 1 AktG systematisch ausschließlich bei der AG verortet, so dass im personellen Schutzbereich bereits an dieser Stelle eine Analogie der Norm erwogen werden müsste. Dann stellt sich aber die Folgefrage, weshalb bei Bejahung einer analogen Anwendung des $\$ 117$ Abs. 1 AktG auf andere Gesellschaftsformen eine Schutzgesetzeigenschaft noch diskutiert werden sollte, böte sodann $\mathbb{\$} 117$ Abs. 1 AktG doch schon selbst die Anspruchsgrundlage.

45 Vgl. Larenz/Canaris, Lehrbuch des Schuldrechts, 13. Aufl., 1994, Band II/2, § 77 I 1 c; Hager, aaO (Fn. 23), $\$ 823$ BGB Rdn. G 4; Schiemann, in: Erman, BGB, 14. Aufl., 2014, $₫ 823 \mathrm{Rdn} .153$.

46 Vgl. BGHZ 66, 388, 390 = NJW 1976, 1740; BGHZ 84, 312, 314 = NJW 1982, 2780 f; BGHZ 100, $13 \mathrm{ff}=$ NJW 1987, 983. Ist der Individualschutz bloß reflexartig vermittelt, so ist Schutzgesetzeigenschaft zu verneinen, vgl. BGHZ 89, 383, $400 \mathrm{f}=\mathrm{NJW} 1984$, 1226, 1230; BGHZ 100, $13 \mathrm{ff}=$ NJW 1987, 983; BGH NJW 2006, 2110. S. a. zu den Grundüberlegungen K. SChmidt, FS Zeuner, 1994, 259; DERs., JuS 1993, 985, 986 f.

47 Vgl. Katzenmeier, aaO (Fn. 29), \8 823 BGB Rdn. 530 m. w. N.

48 Katzenmeier, aaO (Fn. 29), auch modale Schutzbereich genannt, vgl. Wagner, aaO (Fn. 7), $₫ 823$ BGB Rdn. 418.

49 Spindler, Münchener Komm. z. AktG, 14. Aufl., 2014, $\$ 117$ Rdn. 87; Hürfer, AktG, 11. Aufl., 2014, $\$ 117$ Rdn. 14; Kort, in: Hopt/Wiedemann, AktG, 4. Aufl., Lieferung 24, 2011, \117 Rdn. 256; Mertens/Cahn, Kölner Komm. z. AktG, Band II, 3. Aufl., 2012, \$117 Rdn. 48. A. A. wohl BrüggemeIER, AG 1988, $93,97$. 


\section{b) Das Gefüge des GmbHGes}

In den Vorschriften des GmbHGes finden sich ebenfalls keine geeigneten Ansätze, die in Bezug auf Außenstehende schutzgesetztauglich wären. So können die Vorschriften über die innere Kompetenzordnung der $\$ \$ 37$ Abs. 1 und $45 \mathrm{ff} \mathrm{GmbHG}$ ausschließlich die Gesellschafter und Geschäftsführer (bei Existenz im Einzelfall auch die Aufsichtsratsmitglieder und Gesellschafter) binden ${ }^{50}$. Aus diesen Vorschriften ergibt sich jedoch nicht, dass eine dritte Person, die sich in Gesellschaftsangelegenheiten einmischt und dieses Gefüge stört, deliktisch haften müsste ${ }^{51}$. Die Haftung des Geschäftsführers nach $\ 43$ Abs. 2 GmbHG bildet personell einen gesellschaftsrechtlichen Sondertatbestand und kann auf Dritte ebenfalls nicht ausgedehnt werden ${ }^{52}$.

\section{c) Besonderes Wirtschaftsdeliktsrecht}

Die $\mathbb{S} 16$ ff UWG böten zwar Schutzgesetzeigenschaft ${ }^{53}$, jedoch bedürfte es hierfür der in den Tatbeständen genannten qualifizierten unlauteren Wettbewerbshandlungen. Werden also im Einzelfall durch den Dritten Verwaltungsorgane zum Geheimnisverrat verleitet, so steht eine Haftung nach $\ 823$ Abs. 2 BGB außer Frage. Auch kann ein Verstoß gegen die $\$ \$ 16 \mathrm{ff} U W G$ über $\$ 3$ Abs. 1 UWG verfolgt werden ${ }^{54}$. Bei den vorliegend interessierenden Konstellationen können die $\mathbb{\int} \mathbb{1} 16 \mathrm{ff}$ UWG jedoch nicht weiterhelfen.

\section{d) Ansatz über das Strafrecht}

Im Falle der Einflussnahme durch Bestechung im geschäftlichen Verkehr zwecks Verzerrung des Wettbewerbs nach $\$ 299$ Abs. 2 StGB ist eine Haftung

50 Bpsw. Verstöße des Geschäftsführers lösen ausschließlich die vorgesehenen gesellschaftsrechtlichen Tatbestände, etwa $\ 43$ Abs. 2 GmbHG aus, vgl. Sснміdт, in: Ensthaler/Füller/Schmidt, GmbHG, 2. Aufl., 2009, \$37 Rdn. 10; HeIndL, in: Heybrock, Praxiskomm. z. GmbH-Recht, 2010, $\$ 37$ Rdn. 42 ff, 47. Daraus ergibt sich, dass es sich nicht um Schutzgesetze i. S. d. $\$ 823$ Abs. 2 BGB handeln kann.

51 Geregelt ist ausschließlich das interne Gefüge, ohne dass hierdurch Institutionenschutz vermittelt würde, vgl. BGHZ 62, 166, 169. Vielmehr verteidigen diese grundsätzlich dispositiven Regelungen letztlich den Kern der Gesellschafterkompetenzen und weisen im Zusammenspiel mit der jeweiligen Satzung die Richtung der Gesellschaftsleitung, Schmidt, aaO (Fn. 50), $\$ 46$ GmbHG Rdn. 1.

52 Vgl. BGHZ 125, 366, 375; BGHZ 110, 342, 360.

53 Allg. M. vgl. nur BornKamm, in: Hefermehl/Köhler/Bornkamm, UWG, 33. Aufl., 2015, $\ 16$ Rdn. 29. Näher zur Diskussion der Nutzbarmachung des $\$ 823$ Abs. 2 BGB im besonderen Wettbewerbsrecht K. SснміDT, FS Schwark, 2009, S. 753 ff, $768 \mathrm{f}$.

54 Bornkamm, aaO (Fn. 53), $\mathbb{1} 16$ UWG Rdn. 29. 
des Einflussnehmers über $₫ 823$ Abs. 2 BGB unzweifelhaft gegeben ${ }^{55}$, jedoch ist hiermit nur ein Extremfall der Einflussnahme abgedeckt, welcher die der Diskussion zu Grunde liegenden Sachverhaltskonstellationen nicht zu erfassen vermag. Ebenfalls nicht zu bestreiten ist eine Haftung nach $₫ 823$ Abs. 2 BGB, sofern der Einflussnehmer mit Bereicherungsabsicht das jeweilige Verwaltungsorgan vorsätzlich täuscht und mittels einer so erfolgten Irrtumserregung einen Schaden der Gesellschaft durch selbstschädigende Vermögensverfügung erreicht, $\$ 263$ Abs. $1 \mathrm{StGB}^{56}$. In den hier besprochenen Konstellationen fehlt es aber bereits regelmäßig an der Täuschung, jedenfalls aber an einer stoffgleichen Bereicherungsabsicht ${ }^{57}$, da der Einflussnehmer gerade nicht die Absicht verfolgt, sich oder einen Dritten mit den Vermögensabflüssen der Risikogeschäfte zu bereichern.

Des Weiteren ist eine Haftung als Täter (unmittelbar wie mittelbar) nach den $\$ \int 823$ Abs. 2 BGB i. V.m. 266 Abs. 1 StGB mangels Vermögensbetreuungspflicht ${ }^{58}$ des Außenstehenden nicht zu erwägen ${ }^{59}$. Allerdings kommt eine Haftung als Anstifter oder Gehilfe der Untreuetat nach $\$ 830$ Abs. 1 Satz 1, Abs. 2 BGB in Betracht, sofern eine teilnahmefähige Haupttat festgestellt werden kann.

Es ist unstreitig, dass der GmbH-Geschäftsführer einer Vermögensbetreuungspflicht für die Gesellschaft i.S. d. $\$ 266$ Abs. 1 StGB unterliegt ${ }^{60}$. Handelt der Geschäftsführer pflichtwidrig, resultiert hieraus ein Vermögensnachteil und nimmt er beides vorab unter Erkennung des Risikos billigend in Kauf, so kommt Untreuestrafbarkeit in Betracht ${ }^{61}$. Dieser weite Tatbestand brachte in der Vergangenheit bereits mehrfach die Frage hervor, ob hierdurch jedem Verwaltungsmitglied strafrechtliche Verantwortlichkeit droht, wenn pflichtwidrig unternehmerische Risiken eingegangen werden ${ }^{62}$. Es wurde erkannt, dass $\$ 266$ Abs. 1 StGB nicht dazu geschaffen sein kann, die Basis für eine

55 Vgl. Heine, in: Schönke/Schröder, StGB, 29. Aufl., 2014, \299 Rdn. 20; Wagner, aaO (Fn. 7), \823 BGB Rdn. 423.

$56 \$ 263$ StGB umfasst personell und sachlich jedenfalls den deliktischen Schutz dessen, der einen Vermögensnachteil durch Selbst- oder zurechenbare Drittschädigung erleidet, allg. M. vgl. nur BGHZ 57, 137 = NJW 1972, 36.

$57 \mathrm{Zu}$ den spezifischen Anforderungen an die stoffgleiche Bereicherungsabsicht, vgl. SATZGER, in: Satzger/Schluckebier/Widmaier, StGB, 2. Aufl., 2014, \$263 Rdn. $291 \mathrm{ff}, 295 \mathrm{ff}$ m. w. N.

58 Vgl. BGHSt 24, 286 f; Fischer, aaO (Fn. 40), $\$ 266$ StGB Rdn. 21 m. w. N.

$59 \rrbracket 266$ StGB ist ein Sonderdelikt, so dass weder unmittelbare noch mittelbare Täterschaft in Betracht kommen, sofern keine Vermögensbetreuungspflicht in der Person des Handelnden besteht, vgl. BGHSt 13, 330; OLG München, JZ 1977, 411.

60 Vgl. BGHSt 34, 379, 387; BGH NStZ 1995, 185 f; SEIER, in: Achenbach/Ransiek/Rönnau, Hdb. des Wirtschaftsstrafrechts, 4. Aufl., 2015, Rdn. $284 \mathrm{f}$.

61 BGHSt 34, 379, 387; BGH NStZ 1995, 185 f.

62 Drohende „Lähmung des Wirtschaftslebens“, vgl. SALIGER, in: Satzger/Schluckebier/ Widmaier, StGB, 2. Aufl., 2014, \$266 Rdn. 47. 
strafgerichtliche Überprüfung aller riskanten Unternehmensentscheidungen zu bieten ${ }^{63}$.

aa) Zunächst ist umstritten, ab welchem Moment ein Risikogeschäft eine untreuerelevante Pflichtverletzung darstell $t^{64}$. Jedenfalls Einigkeit dürfte heute darüber zu erzielen sein, dass ein Risiko, welches sich im Bereich der Business Judgement Rule (BJR - $\$ 93$ Abs. 1 Satz 2 AktG) bewegt, mangels Missbrauch der treuhänderischen Stellung jedenfalls dann $\$ 266$ Abs. 1 StGB nicht begründen kann, wenn ein klares Verbot zum Abschluss des jeweiligen Rechtsgeschäfts nicht ersichtlich ist ${ }^{65}$.

Außerhalb des „safe harbour" ${ }^{66}$ soll die Abgrenzung danach erfolgen, ob das Risiko sich gleichsam als ein Verhalten des Treuhänders „nach Art eines Spie-

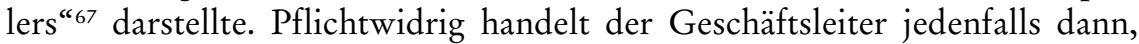
wenn er einen vom Geschäftsherrn vorgegebenen „Risikokorridor“" verlässt ${ }^{68}$. Hierdurch wird jedoch noch keine Aussage über die billigende Inkaufnahme einer Gesellschaftsschädigung getroffen ${ }^{69}$. Fehlt es an einer Vorgabe für riskante Geschäfte, so ist der Treuhänder gehalten, den mutmaßlichen Willen bestmöglich vor dem Hintergrund von Art und Zweck der Geschäftslage und Branchenüblichkeit zu ermitteln ${ }^{70}$. Die entsprechende Überschreitung muss der Geschäftsführer zusätzlich erkannt oder sich dieser Erkenntnis zielgerichtet verschlossen haben, wobei die genauen Anforderungen an den Tätervorsatz umstritten sind (s. u. bb) ${ }^{71}$. Bereits hieraus folgt eine erhebliche Einschränkung für die untersuchten Konstellationen, da insbesondere jene Fälle nicht umfasst werden, in denen nach einer ungenauen oder fehlerbehafteten Abwägung der Geschäftsführer der GmbH jedenfalls nicht daran dachte, gleichsam spielerisch Risiken einzugehen. Zudem verbleibt eine weitreichende Grauzone der Abwägung, in welcher der Geschäftsführer sich damit verteidigen kann,

63 Vgl. Anders, Untreue zum Nachteil der GmbH, 2012, S. 35 ff, $94 \mathrm{ff}$.

64 Zum Streitstand SAliger, aaO (Fn. 62), \$266 StGB Rdn. 47 m. w. N.

65 Basierend auf BGHZ 135, 244 in $\$ 93$ Abs. 1 Satz 2 AktG durch das UMAG vom 22. 09. 2005, BGBL. I Nr. 60, S. 2802 inkorporiert. Zum Ansatz über den Vermögensnachteil, aber noch ohne klare Einbeziehung der Business Judgement Rule (BJR) s. BGH StV 2004, 424 f; Nelles, Untreue zum Nachteil von Gesellschaften, 1991, S. 565 ff; Hillenkamp, NStZ 1981, 164 ff; Bringewat, JZ 1977, 669.

66 Vgl. BGH NZG 2011, 1271; Peters, AG 2010, 811; Roth, Unternehmerisches Ermessen und Haftung des Vorstands, 2001, S. 105 f.

67 So schon RG JW 1935, 2639; vgl. dann später BGH NJW 1975, 1235 f; NJW 1990, 3220; aus der Lit. Fischer, aaO (Fn. 40), \$266 StGB Rdn. 67; Esser, Anwaltskomm. StGB, 2. Aufl., 2015, $\$ 266$ Rdn. 129.

68 Vgl. Hillenkamp, NStZ 1981, 164, $166 \mathrm{f}$.

69 Vgl. Saliger, aaO (Fn. 62), $\$ 266$ StGB Rdn. 48 f m. w. N.

70 BGH StV 2004, 424; BGH NJW 1975, 1234, 1236; WAssmer, Untreue bei Risikogeschäften, 1998, S. 28; Rose, wistra 2005, $286 \mathrm{f}$.

71 Vgl. den Ansatz des 2. Strafsenats in BGHSt 5, 100, 121; BGH NStZ 2007, $704 \mathrm{f}$ gegenüber der ablehnenden Haltung des 1. Strafsenats, s. BGH wistra 2008, 343 ff. 
dass zwar ein hohes Risiko erkannt wurde, dennoch dasselbe - möglicherweise unter Verkennung von Branchenüblichkeiten und/oder Gesellschafterwillen - nicht als übermäßig eingestuft wurde.

bb) Daneben sind die mit den vorangegangenen Ausführungen verquickten Einschränkungen der Rechtsprechung zur gebotenen Verengung des Untreuetatbestandes bedeutsam. Diese greifen auf der Ebene des Vorsatzes und gliedern sich in eine differenzierte Betrachtung von Pflichtwidrigkeit und Vermögensnachteil ${ }^{72}$ sowie in erhöhte Anforderungen an die Feststellung vorsätzlichen Handelns, insbesondere wenn „nur“ bedingter Vorsatz im Raum steht ${ }^{73}$. Diese Eingrenzungsbestrebungen werden mit Hinweis auf ihre Unbestimmtheit und mangelnde Praktikabilität scharf kritisiert, wobei die h. M. in der Literatur für Begrenzungen im objektiven Tatbestand des $\$ 266$ Abs. $1 \mathrm{StGB}$ eintritt ${ }^{74}$. Folgt man dem Ansatz des BVerfGs sowie der Linie des 2. Strafsenats des BGH, so wird die teleologische und zugleich vor dem Hintergrund von Art. 103 Abs. 1 GG verfassungsrechtlich gebotene Reduktion des Tatbestandes wirksam erreicht, indem eine Einbeziehung der Schadensrealisierung in den Tätervorsatz gefordert wird ${ }^{75}$. Bei Annahme dieser Restriktion schiede in den untersuchten Konstellationen eine Untreuestrafbarkeit aus, da der Geschäftsführer gerade im Sinne bewusster Fahrlässigkeit auf einen guten Ausgang vertraut und den risikobedingt drohenden Vermögensabfluss nicht billigt.

cc) Die wesentliche Einschränkung für den Untreuetatbestand ergibt sich jedoch aus der Leitentscheidung des BVerfGs aus dem Jahre 2010, in dessen Rahmen die Untreuestrafbarkeit nur bei evidenten und dementsprechend schweren Pflichtverletzungen zu bejahen sei $^{76}$. Diese Entscheidung führt gerade in den hier erörterten Konstellationen häufig zur Verneinung des Tatbestandes, da jedenfalls ohne klare Vorgaben von Satzung oder Gesellschaftergremium eine Pflichtwidrigkeit nicht evident sein wird. Das gilt vor allem für Risikoentscheidungen. Die Auswirkungen der Entscheidung waren jüngst beim LG Hamburg in der Rechtssache HSH-Nordbank zu spüren ${ }^{77}$. Als Synthese obiger Erörterung kann festgehalten werden, dass auch der Untreuetatbestand weder dazu geschaffen ist noch praktikabel erscheint, über $\mathbb{8} 823$

72 Vgl. BGH NStZ 2013, 715.

73 Vgl. BGH NJW 1975, 1234, 1236.

74 Vgl. Schünemann, Leipziger Komm. z. StGB, 12. Aufl., 2012, \$266 Rdn. 190; DierLAmm, Münchener Komm. z. StGB, 2. Aufl., 2014, \$266 Rdn. 238; Perron, in: Schönke/Schröder, StGB, 29. Aufl., 2014, \266, Rdn. 50; Kubiciel, NStZ 2005, 356; Seier, aaO (Fn. 60), Rdn. 86; SAlinger, HRRS 2006, 23.

75 Vgl. BVerfG NJW 2009, 2370, 2373; BGHSt 51, 100, 121. Ablehnend BGH NJW 2008, $2451 \mathrm{f}$ (1. Strafsenat).

76 Vgl. BVerfGE 126, 170 = NJW 2010, 3209. Hier ist das BVerfG bemüht, der Idee der Literatur (vgl. Fn. 74) zur Eingrenzung von $\$ 266$ Abs. 1 StGB innerhalb des objektiven Tatbestandes zu folgen.

77 Vgl. LG Hamburg, Urt. v. 09. 07. 2014, Az. 608 KLs 12/11, 5550 Js 4/09. 
Abs. 2 BGB die Haftungsfälle externer Einflussnahme hinreichend zu erfassen.

\section{6. $\int 826 B G B-$ „Der schwarze Ritter"78}

Der Schutz des primären Vermögens gegen jedermann ist bei Vorliegen der Voraussetzungen des $\$ 826$ BGB gegeben ${ }^{79}$. Hierfür bedarf es im Einzelfall einer vorsätzlichen, sittenwidrigen Schädigung. Das Vorsatzmoment ist dabei durch die Rechtsprechung stark aufgeweicht worden ${ }^{80}$ und heute nicht mehr die entscheidende Hürde. Vielmehr gilt es, besondere sittenwidrigkeitsbegründende Umstände darzulegen, was zu einer Fallgruppensystematik geführt hat ${ }^{81}$, die in der Rechtspraxis wesentlich an die Stelle des Versuchs einer universell anerkennungsfähigen, positiven Begriffsbestimmung der Sittenwidrigkeit getreten ist $^{82}$. Sittenwidrigkeit wurde von Deutsch auch trefflich als „qualifizierte Form der Rechtswidrigkeit mit subjektiver Färbung "83 beschrieben.

Eine spezielle Fallgruppe für die rechtswidrige Einflussnahme Externer auf die Entscheidungsträger in der Gesellschaft ist bislang nicht anerkannt. Allerdings können Wertungsgesichtspunkte und ähnliche Entscheidungslinien für die hier interessierenden Konstellationen herausgearbeitet werden.

\section{a) Keine Vergleichbarkeit mit dem räuberischen Aktionär}

Aus dem gesellschaftsrechtlichen Sektor erscheint zunächst das Vorgehen räuberischer Aktionäre im Wege der Anfechtungsklage nach $\$ 246$ AktG als naheliegend ${ }^{84}$, da hier jedenfalls $\ 826$ BGB unmittelbar durch die Gesellschaft geltend gemacht werden kann, während in anderen Fällen Dritte, insbeson-

78 So trefflich bezeichnet von Förster, AcP 209 (2009), 398 für die Anwendung im Gesellschaftsrecht.

79 Vgl. Deutsch, Haftungsrecht I, 1976, Rdn. 67; Larenz/Canaris, aaO (Fn. 45), \$ 78 I 1, S. 447; $\mathbb{} 78$ II 2, S. 452.

80 Ausführlich Wagner, aaO (Fn. 7), $\$ 826$ BGB Rdn. $24 \mathrm{ff}$, insbesondere $29 \mathrm{ff} \mathrm{m}$. w. N.

81 Ausführlich Wagner, aaO (Fn. 7), $\$ 826$ BGB Rdn. $54 \mathrm{ff}$.

82 Näher zum Sittenwidrigkeitsverdikt SACK, WRP 1985, 1 ff; WAGNER, aaO (Fn. 7), \8 826 BGB Rdn. 8 ff; Oechsler, in: Staudinger, BGB, 2014, $\$ 826$ Rdn. $24 \mathrm{ff}$ speziell zum Bereich des Gesellschaftsrechts Förster, AcP 209 (2009), $398 \mathrm{ff}$.

83 Deutsch, aaO (Fn. 79), S. 232. Vgl. hierzu auch die eingehende Analyse von SACK, WRP 1985, $1 \mathrm{ff}$ mit erheblicher Kritik an der h. M., wobei sich SACK im Wesentlichen mit seinen Einwänden auf Historie und Begrifflichkeit konzentriert sowie die Anstandsformel beleuchtet. Das inhaltliche Argument des damit möglicherweise übersteigerten primären Vermögensschutzes wird in der Kritik nicht adäquat gewürdigt, vgl. SACK, aaO, S. $14 \mathrm{ff}$. 
dere Gesellschaftsgläubiger als Anspruchsinhaber auftreten ${ }^{85}$. Allerdings besteht der Vorwurf gegen entsprechend handelnde Aktionäre gerade darin, dass sie ihre gesellschaftsrechtliche Treuepflicht qualifiziert aus Eigennutz verletzen und Mitgliedschaftsrechte hierfür missbrauchen, die auch Minderheitenschutz vermitteln sollen ${ }^{86}$. Dies kann einer externen Person ohne Gesellschaftsrechte nicht vorgeworfen werden. Die Position der jeweiligen Schädiger ist in Bezug auf die Art und Weise der Nähebeziehung zur Gesellschaft nicht vergleichbar.

\section{b) Existenzvernichtender Eingriff - Tribotel-Doktrin ${ }^{87}$}

Eine weitere Fallgruppe, die scheinbar den Konstellationen der rechtswidrigen Einflussnahme nahe steht, ist der existenzvernichtende Eingriff durch einen Gesellschafter $^{88}$. Der BGH nimmt eine Haftung des Gesellschafters nach $\ 826$ BGB gegenüber der Gesellschaft (reine Innenhaftung) unter Durchbrechung des gesellschaftsrechtlichen Trennungsprinzips an, „wenn er auf die Zweckbindung des Gesellschaftsvermögens keine Rücksicht nimmt und der Gesellschaft ohne angemessenen Ausgleich - offen oder verdeckt - Vermögenswerte entzieht, die sie zur Erfüllung ihrer Verbindlichkeiten benötigt" ${ }^{\text {"89 }}$.

Diese Entziehung von Vermögen ist zwar ohne Weiteres im Wege rechtswidriger Einflussnahme über die Brücke des pflichtwidrigen Handelns eines Verwaltungsorgans denkbar, jedoch sind Schutzzweck dieser Haftung und somit auch Eingreifen nicht mit den hier erörterten Konstellationen vergleichbar. Die Haftung für einen existenzvernichtenden Eingriff zielt auf den Schutz der Gesellschaftsgläubiger und bildet daher eine Haftung wegen Insolvenzverursachung oder -vertiefung ${ }^{90}$. Die Haftung steht nicht der Gesellschaft zum Erhalt ihrer Vermögensinteressen und des damit verbundenen Shareholdervalue zu. Sie wird dementsprechend auch bei Insolvenzverfahrenseröffnung ausschließlich durch den Insolvenzverwalter geltend gemacht ${ }^{91}$.

84 S. BGHZ 107, 296 = NJW 1989, 2689, 2691 ff; Förster, AcP 209 (2009), 403 f; HüFFER, aaO (Fn. 49), $\$ 245$ AktG Rdn. $22 \mathrm{ff}$.

85 Vgl. die Übersicht bei Schaub, PWW BGB, 10. Aufl., 2015, $\$ 826$ Rdn. 14 ff; KatZenMEIER, aaO (Fn. 29), $\$ 826$ BGB Rdn. 18 ff.

86 So BGHZ 107, 296 = NJW 1989, 2689, $2691 \mathrm{ff}$.

87 Vgl. BGHZ 173, 246 = NJW 2007, 2689 seither st. Rspr. vgl. die Übersicht bei WaGneR, aaO (Fn. 7), $\$ 826$ BGB Rdn. 134 Fn. 575.

$88 \mathrm{Zu}$ den Anfängen vgl. K. Sснмidt, NJW 2001, 3577 ff. Übersicht bei Wagner, aaO (Fn. 7), \826 BGB Rdn. $132 \mathrm{ff}$.

89 BGHZ 173, 246, 252 Rdn. 18 = NJW 2007, 2689.

90 Vgl. BGHZ 179, 344 Rdn. 16, 22 = NJW 2009, 2127; BGH ZIP 2012, 1071 Rdn. 13.

91 BGHZ 164, 50, 62 f = NJW 2005, 3137; BGH NJW 2006, 1344, 1345 Rdn. 10; Weller, DStR 2007, 1166 f; HABERSACK, ZGR 2008, 533, 548; s. a. BGH NJW 2005, 3137, $3141=$ ZIP 2005, $1734 \mathrm{f}$. 
Sofern gleichwohl der Haftungstatbestand nach $₫ 826$ BGB auf Basis dieser Fallgruppe eingreift und zugleich eine Konstellation rechtswidriger Einflussnahme vorliegt, handelt es sich um eine zufällige Koinzidenz. Eine Berücksichtigung der Einflussnahme selbst wird jedoch kaum noch erfolgen, da der Wertungsschwerpunkt der zugleich das Sittenwidrigkeitsurteil erlaubt, immer im Bereich der Insolvenzverursachung oder -vertiefung liegen wird.

\section{c) Verleiten zum Vertragsbruch}

Erwägenswert ist auch die Fallgruppe des Verleitens zum Vertragsbruch. Eine Haftung nach $₫ 826$ BGB soll dann in Betracht kommen, wenn der Dritte den Schuldner zum Vertragsbruch verleitet, indem Freistellung von Ansprüchen in Aussicht gestellt wird (erste Fallgruppe) ${ }^{92}$, oder Dritter und Schuldner kollusiv gegen den Gläubiger zusammenwirken (zweite Fallgruppe) ${ }^{93}$ oder der Schuldner besondere Treuepflichten durch den Vertragsbruch verletzt und der Dritte dies weiß (dritte Fallgruppe) ${ }^{94}$.

\section{aa) Freistellen von Haftung}

Die erste Fallgruppe des Anbietens einer Freistellung gegenüber dem Gläubiger des gebrochenen Vertrages geht in der Rechtsprechung des BGH auf eine Konstellation zurück, in der die Bundesrepublik Deutschland (Beklagte) der Verkäuferin eines Grundstücks anbot, eben jenen Vertrag erneut mit der BRD abzuschließen, das Entgelt sofort zu erhalten und vor Schadensersatzansprüchen der Erstkäufer (Kläger) abgesichert zu werden. Hinzu kam, dass die BRD als Beklagte zugleich mit den Klägern über ein Letzteren gehörendes Grundstück an anderer Stelle in kaufvertraglicher Beziehung stand, wobei die Durchführung des Vertrages vom Erwerb des nunmehr mit einem Doppelgebot betroffenen Grundstücks abhängen sollte. Der BGH erkannte in diesem Fall sittenwidrigkeitsbegründende Umstände aus einer grundsätzlich anzustellenden Gesamtwürdigung aller Aspekte des Einzelfalls, wobei die Begründung maßgeblich auf die Kombination aus Freistellungsversprechen und Handeln der öffentlichen Hand gestützt wurde. Die BRD hatte ihre einflussreiche Position gerade in Bezug auf die Verkäuferin genutzt, indem sie zielgerichtet jegliche Handlungsleitung des Zivilrechts ausschaltete, die die Verkäuferin zur Beachtung des Grundsatzes pacta sunt servanda hätten bewegen können. Dies geschah im Übrigen auch ausschließlich zur Verfolgung eigener Marktinteres-

92 Vgl. BGH NJW 1981, $2184 \mathrm{f}$.

93 Vgl. BGHZ 14, 313, $317=$ NJW 1954, $1925 \mathrm{f}$.

94 Vgl. BGHZ 12, 308, 320 = NJW 1954, $1159 \mathrm{f}$. 
sen, die sich gerade als geeignet erwiesen, die Geschädigten wirtschaftlich zu treffen ${ }^{95}$.

Es zeigt sich, dass die verkürzte Darstellung der ersten Fallgruppe auf ein Schlagwort missverständlich sein kann ${ }^{96}$. Der 5. Zivilsenat hatte seiner Zeit dem Umstand Bedeutung beigemessen, dass Dritter die öffentliche Hand war, die im Rahmen fiskalischen Handelns bei der Teilnahme am privaten Wirtschaftsverkehr rücksichtsvoll vorzugehen habe ${ }^{97}$. Zudem fielen die Begleitumstände ins Gewicht, wonach die BRD gerade auch gehandelt hatte, um den Klägern ihre Verhandlungsposition in anderer Angelegenheit zu nehmen oder jedenfalls abzuwerten ${ }^{98}$. Bezüglich der Situation der Einflussnahme bei Gesellschaftsentscheidungen sind aber zusätzlich zwei Elemente hervorzuheben. Hält man die Rechtsprechung zur Verleitung zum Vertragsbruch in diesen Fällen grundsätzlich für anwendbar ${ }^{99}$, so wird es regelmäßig nicht dazu kommen, dass der Einflussnehmer Haftungsfreistellung verspricht, da dieses Risiko kaum tragbar und auch sicherlich nicht gewollt wäre. Auch zielt der Vertragsbruch in den hier untersuchten Fällen häufig auf die Durchsetzung einer bestimmten Meinung in Bezug auf eine gute Unternehmensführung.

\section{bb) Kollusion}

Die zweite Konstellation des kollusiven Zusammenwirkens zum Nachteil des Vertragspartners ${ }^{100}$ muss nicht näher am Fall beleuchtet werden, da diese bei der hier diskutierten Einflussnahme a priori nicht greifen kann. Selbstverständlich ist es denkbar, dass ein Dritter in kollusivem Zusammenwirken mit einem Verwaltungsmitglied der Gesellschaft einen Schaden zufügt. Auch leuchtet es ein, in diesen Fällen sittenwidrigkeitsbegründende Umstände gerade darin zu erblicken, dass der Externe und das Verwaltungsmitglied sich gegen die Interessen der Gesellschaft verschwören und die gegebenen gesellschaftsrechtlichen Einflussnahmemöglichkeiten des Verwaltungsmitglieds hierfür zielgerichtet missbrauchen. Allerdings kann hiermit keine Aussage darüber getroffen werden, ob unterhalb genannter Schwelle der Vorwerfbarkeit des deliktischen Verhaltens

95 Vgl. BGH NJW 1981, $2184 \mathrm{f}$.

96 So vielfach in der Kommentarliteratur, vgl. Katzenmeier, aaO (Fn. 29), $\$ 826$ BGB Rdn. 24; Sprau, aaO (Fn. 2), \826 BGB Rdn. 23; Wagner, aaO (Fn. 7), \826 BGB Rdn. 60. Natürlich weist die Literatur zutreffend gleichfalls darauf hin, dass immer eine Gesamtabwägung aller Umstände zu erfolgen habe, die das Urteil eines schwerwiegenden Verstoßes gegen das Grundbedürfnis loyaler Rechtsgesinnung zulasse, vgl. etwa Katzenmeier, aaO, Rdn. 23. Nur so darf letztlich aber auch die Fallgruppensystematik gelesen werden, da es andernfalls zu verfälschten Ergebnissen kommen kann. BGH NJW 1981, 2184, 2186; hierzu Wagner, aaO (Fn.7), \$823 BGB Rdn. 61 mit gebotenen Hinweisen auf ein einschränkendes Verständnis dieses Judikats.

98 BGH NJW 1981, 2184, 2186.

99 So offensichtlich ohne jeden Vorbehalt BGHZ 12, 308, $320=$ NJW 1954, $1159 \mathrm{f}$.

100 BGHZ 14, 313, 317. 
ebenfalls eine Haftung greifen soll, sei diese aus $₫ 826$ BGB oder aus einer anderen Vorschrift begründet. Es handelt sich vielmehr um einen klassischen „Extremfall“101, der die „einzige jedermann einleuchtende Grenze“ ${ }^{102}$ bildet, die die Haftung nach $\$ 826$ BGB nach sich zieht.

\section{cc) Bruch besonderer Trenepflichten}

Scheinbar geradezu auf das Gesellschaftsrecht zugeschnitten, da an einer gesellschaftsrechtlichen Konstellation entwickelt, ist jedoch Variante drei. Die Kläger schlossen mit einer Filmherstellergesellschaft einen Vertrag über die Erstellung eines Dokumentarfilms und beteiligten sich partiell an den Kosten. Der Beklagte gewährte der Herstellergesellschaft später Kredite und ließ sich zur Sicherheit die Verleihrechte am fertigen Film abtreten. Dies geschah in Kenntnis der Kosten- sowie der Gewinnbeteiligung der Kläger ${ }^{103}$.

Von Bedeutung ist zunächst, dass der BGH hier eine Außen-GbR zwischen den Klägern und der Herstellergesellschaft verneint hat. Daraus folgt für die weiteren Erwägungen, dass Geschädigte letztlich keine Gesellschafter einer nach außen getretenen werbenden Gesellschaft waren, sondern das Gesellschaftsverhältnis als auf die reine Beteiligung an Kosten und Ertrag im Innenverhältnis beschränkt angesehen werden musste. Daraus folgerte der BGH, dass die Abtretung der Verleihrechte zwar den Vertrag zwischen der Herstellergesellschaft und den Klägern verletzte, gleichwohl aber nicht mangels Verfügungsmacht der Herstellergesellschaft unwirksam gewesen sei $^{104}$. Sodann stellt der BGH drei Argumentationslinien heraus, die im Fall sittenwidrigkeitsbegründend sein sollen. Erstens würden gesellschaftsrechtliche Treuepflichtverletzungen schwerer als „einfache“ Vertragsverletzungen bei Austauschverträgen wiegen, was sich aus einer Anlehnung an den Gedanken der Strafbarkeit für Untreue nach $\$ 266$ Abs. 1 StGB ergebe. Zweitens sei dieser letzte wirtschaftlich ernstzunehmende Vermögenswert der Herstellergesellschaft nicht belastet worden, da man einen Kredit für den Gegenstand oder dessen Unterhaltung gebraucht, sondern denselben für Drittinteressen der Herstellergesellschaft zweckentfremdet habe. Drittens sei zu berücksichtigen, dass der erstellte Film, an welchem Rechte vertragswidrig an einen Dritten verfügt wurden, überhaupt erst aus der organisatorischen und finanziellen Mithilfe der Kläger auf Basis des erfolgten Zusammenschlusses habe entstehen können. Daraus folge eine besonders starke und schutzwürdige Position der Kläger, die im Rahmen der Sittenwidrigkeitsbewertung Raum greife ${ }^{105}$. 
Wenn nun in der Literatur auf die Fallgruppe der verletzten Treuepflicht abgestellt wird ${ }^{106}$, so ist ausschließlich eine dieser Argumentationslinien des BGH aufgegriffen. Jedoch wird an keiner Stelle der Entscheidung deutlich, ob ein Absinken unter die Kumulation der Begründungslinien ebenfalls dem Sittenwidrigkeitsurteil genügt hätte. Zugleich muss für die vorliegende Debatte das Augenmerk darauf gerichtet werden, dass der Beklagte, der hier die Herstellergesellschaft zum Vertragsbruch des Gesellschaftsvertrages verleitete, dies durch eigenes, unmittelbares Angebot im Wissen der Situation erreichte. Eine Gleichstellung mit der Beeinflussung eines Verwaltungsorgans, welches sodann erst eine eigenständige geschäftliche Entscheidung trifft, erscheint sowohl unter der Gesamtwürdigung der Sittenwidrigkeitsprüfung als auch unter dem Gesichtspunkt des Schutzzwecks der Norm problematisch.

Ein Moment der Bewertung tritt sodann noch hinzu, welches der hier untersuchten (zumeist externen) Einflussnahme regelmäßig nicht anhaftet, welches demgegenüber aber allen Fallgruppen des Verleitens zum Vertragsbruch innewohnt. Der Vertragsbruch hat immer das Ziel oder wenigstens objektiv die Stoßrichtung, den Einflussnehmer oder einen von diesem gewünschten Dritten mit den Früchten des Vertragsbruchs zu beden$\operatorname{ken}^{107}$.

Als Zwischenfazit bleibt festzuhalten, dass die Fallgruppe der Verleitung zum Vertragsbruch die untersuchten Konstellationen nicht abdecken kann. Gleichwohl ist nicht zu übersehen, dass die Rechtsprechung sich bereits mit unzulässiger Einflussnahme auseinandergesetzt hat und Wertungskriterien erarbeitete, die jedenfalls für die Ausfüllung des Sittenwidrigkeitsbegriffs Geltung beanspruchen. Dabei ist die Aussage hervorzuheben, dass gesellschaftsrechtliche Treuepflichtverletzungen in Anlehnung an den Rechtsgedanken des $\$ 266$ StGB schwerer als „einfache“ wiegen sollen. Den Fallgruppen kann aber keine Aussage in Bezug auf eine generelle Haftung für die Einflussnahme auf Gesellschaften und ihre Organe entnommen werden, da der BGH sich in den genannten Judikaten weder mit der Thematik im Allgemeinen noch über die Brücke des Rechts am Unternehmen oder gar mit $\ 117$ Abs. 1 AktG und dessen Wertungen auseinandergesetzt hat. Dies erstaunt, wird doch der Rechtsgedanke des Verbots unmittelbarer Geltendmachung von Reflexschäden über das allgemeine Deliktsrecht durch Gesell-

106 S. SpIndler, in: Bamberger/Roth, BGB, 36. Aufl., 2013, \826 Rdn. 27; Schaub, aaO (Fn. 85), \826 BGB Rdn. 18; Wagner, aaO (Fn. 7), $₫ 826$ BGB Rdn. 60.

107 So bei BGHZ 12, $308 \mathrm{ff}=$ NJW 1954, 1159 ff; bei BGHZ 14, 313, 317 ist dieses Element in Bezug auf das kollusive Handeln sogar integrativer Bestandteil der Tat; ebenfalls hervorgehoben bei BGH NJW 1981, $2184 \mathrm{f}$. Mit entsprechendem Ansatz offenbar WAGNER, aaO (Fn. 7), \826 BGB Rdn. 63. 
schafter mittels eines Verweises auf die Wertungen des $\$ 117$ AktG abgelehnt ${ }^{108}$. Gleiches gilt für die Heranziehung von $\$ 117 \mathrm{Abs} .7 \mathrm{AktG}$ im Rahmen der Anwendung des $₫ 826 \mathrm{BGB}^{109}$.

\section{d) Deliktische Haftung für erteilten Rat}

Über die bereits angesprochenen vertraglichen Haftungsgrundlagen hinaus (s.o.) $)^{110}$ ist die Geltendmachung von Schadensersatzansprüchen wegen Falschinformation nach $\$ 826$ BGB anerkannt, wenn der Ratgeber wissentlich unrichtig Auskunft gibt ${ }^{111}$. Auch ein leichtfertiger Rat „ins Blaue hinein“ soll genügen, wenn jedenfalls mit einem Schadenseintritt zu rechnen war und der Auskunfterteilende die Wichtigkeit für den Geschädigten erkannt hat oder hätte erkennen müssen ${ }^{112}$.

\section{Zwischenfazit}

Es existieren zahlreiche Haftungstatbestände, jedoch werden die hier untersuchten Konstellationen nur partiell und ohne tatbestandlich klare Abgrenzungslinien erfasst.

\section{Zur analogen Anwendbarkeit des $\int 117$ Abs. 1 Akt G}

Die Lückenschließung im GmbH-Recht durch Vorschriften des AktGs blickt auf eine lange Tradition mit heftigen Auseinandersetzungen um das grundlegende Konzept einer personalistischen $\mathrm{GmbH}$ im Spannungsfeld mit der Annäherung an eine rein kapitalistisch ausgerichtete Gesellschaftsform zurück $^{113}$. Zu Recht weist Fleischer in diesem Zusammenhang darauf hin, dass nur eine vorsichtige Annäherung an jeweils gebotene Lückenschließung durch Heranziehung des Aktienrechts dem beweglichen System der GmbH gerecht wird ${ }^{114}$. Ganz in diesem Sinne wird zur Lösung der aufgezeigten haftungsrechtlichen Problematik die analoge Heranziehung des $\$ 117$ Abs. $1 \mathrm{AktG}$,

108 So BGHZ 94, 55, $58=$ NJW 1985, $1777 \mathrm{f}$.

109 Vgl. BGHZ 129, 136, 160 = NJW 1995, 1739, 1748 ff; hierzu Wagner, aaO (Fn. 7), \826 BGB Rdn. 146.

110 S. o. III.1.

111 So schon RGZ 91, 80 f; 157, 228 f; und nachfolgend BGH NJW 1992, 3167, 3174.

112 Vgl. BGH NJW 1992, 3167, 3174.

113 Hierzu Fleischer, GmbHR 2008, $673 \mathrm{ff}$ m.N. aus der Gesetzgebungsgeschichte.

114 Fleischer, GmbHR 2008, $673 \mathrm{ff}, 678$. 
nicht jedoch die Übernahme des gesamten Haftungskonzepts des $₫ 117 \mathrm{AktG}$ vorgeschlagen und somit eine Teilanalogie ${ }^{115}$ erwogen $^{116}$.

Eine analoge Anwendbarkeit von $₫ 117$ Abs. 1 AktG auf die GmbH setzt eine planwidrige Regelungslücke im Bereich des $\mathrm{GmbH}$ - oder des allgemeinen Haftungsrechts sowie eine Vergleichbarkeit der Interessenlagen voraus (unter 3. ${ }^{117}$. Bevor eine Auseinandersetzung mit diesen erforderlichen Positivkriterien stattfindet, soll die Debatte vorab erwägenswerten Ablehnungsgesichtspunkten gewidmet sein (unter 2.). Soweit ersichtlich, gibt es hierzu keine Stellungnahmen in der Rechtsprechung und nur Ansätze in der Literatur ${ }^{118}$. Vereinzelt finden sich knappe Hinweise bei den Kommentierungen zur Verweisungsnorm des $\$ 52 \mathrm{GmbHG}$, wonach trotz des Wortlauts wohl auch $\$ 117$ $A k t G$ einzubeziehen sei ${ }^{119}$. Teilweise wird auch ohne Begründung darauf verwiesen, dass das allgemeine Deliktsrecht genüge, um alle relevanten Haftungslücken zu füllen ${ }^{120}$. Zur Schaffung einer Diskussionsgrundlage steht am Beginn der Betrachtung eine Darstellung der Haftung nach $\$ 117$ Abs. 1 AktG (unter 1.).

\section{Haftung nach $\int 117 \mathrm{Abs.} 1 \mathrm{Akt} G$}

$\$ 117$ Abs. 1 Satz 1 AktG ordnet die Haftung des Einflussnehmers an, der Einfluss auf ein Verwaltungsmitglied der Gesellschaft besitzt und diese Machtposition vorsätzlich nutzt, um das Verwaltungsmitglied zu einem Verhalten zu bestimmen, das kausal zu einem Schaden der Gesellschaft führt. Anspruchsinhaber ist sodann die Gesellschaft. Die Gläubigerstellung wird in $\$ 117$ Abs. 1 Satz 2 AktG auf die Aktionäre erweitert, sofern kein bloßer Reflexschaden ${ }^{121}$ vorliegt. Letzteres ist der Fall, wenn die Schädigung den

115 Hierzu Fleischer, GmbHR 2008, 673 ff, 676.

116 So ist das Gläubigerverfolgungsrecht in $\$ 117 \mathrm{Abs} .5 \mathrm{AktG}$ nach h. M. nicht in das GmbH-Recht zu übertragen, vgl. Wicke, GmbHG, $\$ 13$ Rdn. 13.

117 Vgl. Zippelius, Juristische Methodenlehre, 2005, S. 64 ff.

118 Für partielle Analogiefähigkeit ScHALl, in: Spindler/Stilz, AktG, 3. Aufl., 2015, $\$ 117$ Rdn. 34. Voigt, Haftung aus Einfluss auf die Aktiengesellschaft, 2004 bietet eine breite Analyse des $\$ 117$ AktG, jedoch wird die Analogiefähigkeit der Norm nicht besprochen. Interessant ist auch die Erörterung bei KonT, aaO (Fn. 49), $\$ 117$ AktG Rdn. $90 \mathrm{ff}, 254 \mathrm{ff}$, der das Verhältnis zur Treuepflicht, zu den $\$ \mathbb{S} 823 \mathrm{ff}$ BGB und zu den konzernrechtlichen Bestimmungen der $\int \mathbb{S} 308 \mathrm{ff} A \mathrm{AtG}$ als diskussionswürdig sieht, eine Verallgemeinerung des Rechtsgedankens in $\$ 117$ Abs. 1 AktG jedoch nicht anspricht. Wagner, aaO (Fn. 7), $\$ 826$ BGB Rdn. 145 ff, 152 streift die Problematik, bietet jedoch keine nähere Auseinandersetzung.

119 Vgl. Scholz/Schneider, aaO (Fn. 42), \52 GmbHG Rdn. $537 \mathrm{ff}$.

120 So Heermann, in: Ulmer/Habersack/Löbbe, GmbHG, 2. Aufl., 2014, \52 Fn. 364.

121 Zu Begriff und Grundsätzen vgl. BGHZ 94, 55, 58 = NJW 1985, 1777; BGHZ 105, 121 = NJW 1988, 2794. 
Aktionär selbst trifft und sich nicht nur in Form der Entwertung seiner Anteile vollzieht ${ }^{122}$.

a) Beeinflusste Verwaltungsmitglieder können sowohl der Vorstand als auch der Aufsichtsrat und Prokuristen sowie Handlungsbevollmächtigte sein. Der Kreis ist somit weit gezogen und nicht auf die Spezifika der AG zugeschnitten, was durch die Einbeziehung von Prokuristen und Handlungsbevollmächtigen i. S. d. $\$ \int 48 \mathrm{ff}$ und $54 \mathrm{HGB}$ verdeutlicht wird ${ }^{123}$. Darüber hinaus kommt eine Erweiterung allerdings nicht in Betracht, um einer Ausuferung der Haftung keinen Vorschub zu leisten ${ }^{124}$. Die formale Rechtsstellung muss zum Zeitpunkt der Einflussnahme und auch zum Zeitpunkt der schädigenden Handlung bestehen ${ }^{125}$.

b) Einflussnehmer kann jedes handlungsfähige Rechtssubjekt sein. Es handelt sich um einen Jedermannsparagraphen im klassisch deliktischen Sinne ${ }^{126}$. In Betracht kommen insbesondere Personen, die aufgrund einer Gesellschafteroder Aufsichtsratsstellung Einfluss ausüben können. Gleichermaßen kann der Tatbestand aber auch durch jeden Dritten - Lieferanten, Arbeitnehmer und sonstige Privatpersonen - erfüllt werden ${ }^{127}$. Da die Funktion des Einflussnehmenden rein für sich genommen zunächst irrelevant ist, kommen zudem Stimmrechtsvertreter in Betracht ${ }^{128}$. Selbst die öffentliche Hand ist tauglicher Schädiger ${ }^{129}$.

$122 \mathrm{Vgl}$. Spindler, aaO (Fn. 49), $₫ 117$ AktG Rdn. $52 \mathrm{ff} \mathrm{m.w.} \mathrm{N.}$

123 Vgl. Kort, aaO (Fn. 49), $\$ 117$ AktG Rdn. 110.

$124 \mathrm{Vgl}$. Kort, aaO (Fn. 49), $\$ 117$ AktG Rdn. 103. Für eine erweiterte Auslegung Timm, WM 1991, 481, $489 \mathrm{ff}$.

125 Vgl. Kort, aaO (Fn. 49), \$117 AktG Rdn. 111.

126 Vgl. Mertens/Cahn, aaO (Fn. 49), $\$ 117$ AktG Rdn. 13 f m. w. N. A. A. Voigt, aaO (Fn. 118), S. $219 \mathrm{ff}$ unter Hinweis auf Systematik, Teleologie und Gesetzgebungsgeschichte. Die Konsequenz erstaunt, arbeitet doch gerade Voigt, aaO, S. 5 ff dezidiert heraus, dass die Gesetzgebungsgeschichte von einer gesellschaftsrechtlichen Qualifikation wegverweist und sich allgemeinen Prinzipien verschrieben hat. Bedeutsamer ist aber der Irrtum bei VoIGT, $\mathbb{\$} 117$ Abs. 1 AktG zeige gerade durch die verlangte Nähe von Drittem und Gesellschaft, dass eine gesellschaftsrechtliche Sonderverbindung zu verlangen sei, die letztlich in der Haftung wegen der Stellung als "Quasi-Organ“" gipfele, s. Voigt, aaO, S. 221 unter Hinweis auf die systematischen Grunderwägungen der S. $62 \mathrm{ff}, 165 \mathrm{ff}$. VoIGT missversteht letztlich den Begriff des Einflusses und der diesem zu Grunde liegenden Machtposition. Diese Position wurde von $\$ 117$ Abs. 1 AktG selbst bei der Schaffung in $\$ 101$ AktG 1937 niemals als Nähe zur Gesellschaft, sondern als Nähe und Einflussnahmemöglichkeit zum jeweiligen Verwaltungsorgan begründet. Nur dort kann es schließlich für die relevante Einflussnahme Nähepositionen geben.

$127 \mathrm{Vgl}$. Kort, aaO (Fn. 49), $\$ 117$ AktG Rdn. $97 \mathrm{ff}, 117 \mathrm{ff}$.

128 Vgl. Spindler, aaO (Fn. 49), \$117 AktG Rdn. 11.

129 Vgl. Timm, FS Semler, 1993, S. 611, 613; Kort, aaO (Fn. 49), $\$ 117$ AktG Rdn. 97 ff m.w. N. 
c) Bedeutsam ist die Bestimmung des Begriffs „Einfluss“. Dieser wird zutreffend funktional aufgefasst, was sich zwingend aus dem Normzweck ergibt $^{130}$. Somit kommt jede Art von Machtstellung und damit verbundener Einflussnahmemöglichkeit in Betracht, also sowohl gesellschaftsrechtlich vermittelte Positionen, wie die Stellung als Großaktionär, als auch rein privat emotionale Konstellationen, wie partnerschaftliche Beziehungen ${ }^{131}$. Nach h.M. ist dementsprechend auch der Tatbestand des $\$ 117$ Abs. 1 AktG zutreffend so zu lesen: „Wer vorsätzlich ein Mitglied des Vorstands oder des Aufsichtsrats, einen Prokuristen oder Handlungsbevollmächtigten beeinflusst, zum Schaden der Gesellschaft zu handeln ..." ${ }^{132}$. Dabei ist es nicht geboten, dass die Einflussnahme allein für sich betrachtet in einer rechtlich unzulässigen Form geschieht ${ }^{133}$, (zur Rechtswidrigkeit der Einflussnahme in der Gesamtschau s. u. (e.)). Freilich kann unter präziser Berücksichtigung des Wortlauts von $\$ 117$ Abs. 1 Satz 1 AktG sowie vor dem Hintergrund systematischer Einordnung bei Beachtung des $\$ 117$ Abs. 7 AktG und auch zur Begrenzung der Haftungsweite argumentiert werden, dass die Einflussposition in Bezug auf die Gesellschaft selbst wirken müsse. Damit würden bei $\$ 117$ Abs. 1 Satz 1 AktG jene Konstellationen ausgeklammert, in denen etwa die emotionalen Einflussmöglichkeiten, die ausschließlich ein Mitglied der Leitungs- und Führungsebene betreffen, durch den Einflussnehmer genutzt werden. Diese Frage kann hier nicht eingehend untersucht werden und bleibt einer eigenständigen wissenschaftlichen Erörterung vorbehalten. Sie ist für die Grundsatzfrage der Analogiefähigkeit von $\$ 117$ Abs. 1 AktG nicht entscheidungserheblich.

d) Ein Ausnutzen des Einflusses findet bereits dadurch statt, dass der Einflussnehmer in Kenntnis seiner Machtposition das betroffene Verwaltungsmitglied zum schädigenden Verhalten verleitet ${ }^{134}$. Hervorzuheben ist hierbei, dass entgegen des missverständlichen Wortlauts in $\$ 117$ Abs. 1 Satz 1 AktG die Ausnutzung des Einflusses nicht das Ziel verfolgen oder das Ergebnis haben muss, dass der Einflussnehmer oder ein Dritter hieraus Vorteile erlangt ${ }^{135}$. Derjenige, der einen Vorteil erhält und auch seinen Vorsatz hierauf gerichtet hatte, wird

130 Vgl. Spindler, aaO (Fn. 49), $\mathbb{1 1 7}$ AktG Rdn.4, 16 f. Insofern zustimmend Voigt, aaO (Fn.118), S. 235 ff, freilich mit starken Beschränkungen wegen des zusätzlich verlangten Tatbestandsmerkmals der Quasi-Organstellung, vgl. S. $221 \mathrm{ff}$.

131 H. M. vgl. Mertens/Cahn, aaO (Fn. 49), \$117 AktG Rdn. 13; Brüggemeier, AG 1988, 93, 95; Wiesner, Münchener Handbuch des Gesellschaftsrecht, Band 4, Aktiengesellschaft, 4. Aufl., 2015, \27 Rdn.2; Schall, aaO (Fn. 118), \117 AktG Rdn.15; Kort, aaO (Fn. 49), \117 AktG Rdn. 126 f; Dagegen Bergmann, ZHR 105 (1938), 1, 23; v. Godin/Wilhelmi, AktG 1937, \$101, Anm. 2.

132 Kort, aaO (Fn. 49), $\mathbb{1} 117$ AktG Rdn. 113.

133 Vgl. Begr. RegE, abgedruckt bei KropfF, AktG, 1965, S. 162.

134 Vgl. Mertens/Cahn, aaO (Fn. 49), $\$ 117$ AktG Rdn. 16.

135 Allg. M. vgl. Kort, aaO (Fn. 49), \117 AktG Rdn. 148; Spindler, aaO (Fn. 49), $\$ 117$ AktG Rdn. 22. 
vielmehr eigenständig über $\$ 117$ Abs. 3 AktG als Nutznießer erfasst. Ein Verleiten liegt vor, wenn der Handlungsentschluss durch den Einfluss des Täters hervorgerufen wird ${ }^{136}$, wobei Mitursächlichkeit genügt ${ }^{137}$. Die Beweisschwierigkeiten seitens der Gesellschaft werden dadurch abgemildert, dass eine tatsächliche Vermutung für eine kausale Beeinflussung anzunehmen ist, wenn das Verwaltungsmitglied nach Einflussnahme handelt ${ }^{138}$. Darüber hinaus wird ein Anscheinsbeweis in Bezug auf die Annahme einer Beeinflussung erwogen, wenn der Einflussnehmer aus einem nachgewiesenen Schaden der Gesellschaft eine stoffgleiche Bereicherung erlangt hat ${ }^{139}$. Diese letztgenannte Beweismaßreduktion erscheint aber zweifelhaft, kann es doch denkbar viele Gründe für das Handeln des Verwaltungsorgans gegeben haben, die unabhängig von einer Einflussnahme den Ausschlag gaben. Ein erlangter Vorteil dürfte erst dann ein sinnvolles Indiz für Rückschlüsse bilden, wenn wenigstens eine geeignete Einflussnahmehandlung nachgewiesen werden kann. Im Übrigen muss aus dieser Einflussnahme sodann kausal der Gesellschaft und/oder den Aktionären ein Schaden entstanden $\operatorname{sein}^{140}$.

e) Nach einhelliger Auffassung verlangt der Unrechtstatbestand des $\$ 117$ Abs. 1 AktG auch das Urteil der Rechtswidrigkeit ${ }^{141}$. Insofern wird aber auf Basis des Normzweckverständnisses eine breite Debatte darüber geführt, ob entweder die Pflichtwidrigkeit des Verhaltens des Beeinflussten die Rechtswidrigkeit der Einflussnahme indiziere ${ }^{142}$ oder die Rechtswidrigkeit gleichsam eines offenen Tatbestandes an Hand einer umfassenden Interessen- und Güterabwägung festzustellen sei ${ }^{143}$ oder ob sogar eine Sonderstellung des Einflussnehmers gleich eines Quasiorgans verlangt werden müsse ${ }^{144}$. Für die Dar-

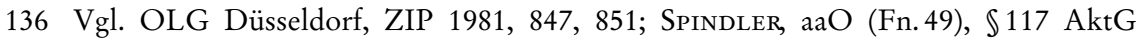
Rdn. 22.

137 Vgl. Kort, aaO (Fn. 49), \$117 AktG Rdn. 144 ff „Kausalnexus“.

138 So zutreffend Spindler, aaO (Fn. 49), $\$ 117$ AktG Rdn. 26. Zustimmend Kort, aaO (Fn. 49), $\mathbb{1 1 7}$ AktG Rdn.146, allerdings m.V.a. frühere Kommentierung KropfF, Münchener Komm. z. AktG, 2. Aufl., 2004, $\$ 117$ Rdn. 23.

139 So noch Kort, aaO (Fn. 49), $\$ 117$ AktG Rdn. 162, jedoch ohne argumentativ unterlegte Begründung.

140 Zur Diskussion um den Schadensbegriff und die Kausalität KorT, aaO (Fn. 49), \$117 AktG Rdn. 134 ff, 147.

141 Allg. M. vgl. Spindler, aaO (Fn. 49), $\$ 117$ AktG Rdn. 31 ff; Kort, aaO (Fn. 49), \$117 AktG Rdn. $37 \mathrm{ff}, 149 \mathrm{ff}$.

142 So i.E. zutreffend Kort, aaO (Fn. 49), \$117 AktG Rdn. 152; BrüggemeIER, AG 1988, 93, 97; Mertens/Cahn, aaO (Fn. 49), $\ 117$ AktG Rdn. 22. A. A. insbesondere Voigt, $\mathrm{aaO}$ (Fn. 118), S. $62 \mathrm{ff}$ an Hand von Normzweckerwägungen die sämtlich darauf basieren, dass $₫ 117 \mathrm{AktG}$ als spezifisch gesellschaftsrechtlicher Tatbestand interpretiert wird, sowohl nach subjektiver wie auch nach objektiver Teleologie der Vorschrift letztlich nicht entspricht.

143 Eine ausführliche Übersicht bietet Voigt, aaO (Fn. 118), S. 71 ff m. w. N.

144 So Voigt, aaO (Fn. 118), S. $81 \mathrm{ff}$. 
stellung des Streitstandes sei auf die Ausführungen bei Voigt $t^{145}$ und Kort verwiesen $^{146}$. Dabei ist der Sichtweise von Kort, der letztlich zu einer Indizierung der Rechtswidrigkeit bei gegebener Tatbestandsmäßigkeit kommt, zuzustimmen. Korts umfangreicher Argumentation ist hinzuzufügen, dass $\$ 117$ Abs. 1 AktG gerade nicht als Rahmenrecht konstruiert wurde, wie dies bei den schwer bestimmbaren Gütern des Rechts am Unternehmen und des allgemeinen Persönlichkeitsrechts der Fall ist. Vielmehr zielt die Norm ihrem insoweit eindeutigen Wortlaut nach darauf ab, den Konnex von Einflussnahme durch Nutzung einer Machstellung zur Bewirkung eines pflichtwidrigen Handelns eines Verwaltungsorgans generell zu unterbinden. Eine Rechtfertigung muss gerade die Ausnahme bilden. Konstruierte man $\ 117$ Abs. 1 AktG als offenen Tatbestand, so wäre es gleichsam der Problematik beim Recht am Unternehmen wiederum Sache des Tatrichters, den Rahmen wirtschaftlich zulässiger Verhaltensweisen herauszuarbeiten, obwohl das Gericht hierzu weder ausgebildet ist noch berufen sein sollte ${ }^{147}$. Freilich muss es Bewegungsspielraum für ungeschriebene Fälle geben, wie dies auch mit den $\$ \$ 826$ BGB und 3 UWG der Fall ist, jedoch ist es primär Sache des Gesetzgebers, einen möglichst präzisen Ordnungsrahmen für den Leistungswettbewerb zu setzen, der nicht darüber aufgeweicht werden darf, dass zivilrechtliche Handlungsvorgaben wie $\$ 117$ Abs. 1 AktG durch Rechtsprechung oder Literatur zu offenen Tatbeständen degradiert ${ }^{148}$ und somit ihrer gebotenen und vom Gesetzgeber avisierten Schärfe beraubt werden ${ }^{149}$. Wenn Voigt bereits in Bezug auf die Diskussion um eine Qualifikation als offener Tatbestand zu bedenken gibt, dass $\ 117 \mathrm{Abs.} 1 \mathrm{AktG}$ letztlich kein Täter-, sondern ein Anstifterdelikt sei und somit Haftungslücken drohten ${ }^{150}$, ist dem nicht zuzustimmen. Voigt weist darauf hin, dass ein getäuschter Geschäftsleiter nach seiner Informationslage pflichtgemäß handele, während der Einflussnehmer gerade durch die geschickte List der Haftung entgehen könne. Dies beruht auf der Annahme, dass der schuldlos irrende Geschäftsleiter objektiv sorgfaltsgemäß handeln würde ${ }^{151}$. Dies darf aber gerade nicht angenommen werden.

145 Voigt, aaO (Fn. 118), S. 62 ff.

146 Kort, aaO (Fn. 49), $\$ 117$ AktG Rdn. 37 ff.

147 Dementsprechend der frühe Grundansatz bei RGZ 3, 68 mit der Erklärung, dass Wettbewerbsverhalten grundsätzlich zulässig sei, solange es nicht gesetzlich verboten werde. Daraus resultierte die Schaffung eines Lauterkeitsrechts mit Generalklausel im Jahre 1896, vgl. WADLE, JuS 1996, 1064.

148 Mit diesem Bestreben scheinbar Wiesner, aaO (Fn. 131), \$27 Rdn. 5; ebenso MerTENs/CAHn, aaO (Fn. 49), \$117 AktG Rdn. 22.

149 In diesem Zusammenhang wird insbesondere mit Blick auf das Recht am Unternehmen und dessen Ausweitung zutreffend darauf hingewiesen, dass jegliche Ausweitung genereller Verhaltenspflichten auf einen Generalschutz fremder primärer Vermögensinteressen hinausliefe, vgl. v. BAR, Verkehrspflichten, 1980, S. 157 ff, 204 ff; BRÜGGEMEIER, Deliktsrecht, 1986, Rdn. 330, $445 \mathrm{ff}$.

150 Voigt, aaO (Fn. 118), S. 45 f.

151 Voigt, aaO (Fn. 118), S. 46. 
Die Erkenntnismöglichkeiten des Geschäftsleiters und dessen Fehlannahmen auf Basis von Dritttäuschungen sind unter dem Gesichtspunkt des Verschuldens - und eben nur dort(!)-zu diskutieren ${ }^{152}$. Ein Bestimmen zur objektiv sorgfaltswidrigen Tat ist gleichwohl jeder Zeit denkbar ${ }^{153}$.

f) Schließlich muss der Einflussnehmer hinsichtlich aller oben genannten Merkmale vorsätzlich handeln. Es genügt dolus eventualis ${ }^{154}$, während, anders als im Rahmen des $₫ 826$ BGB $^{155}$, Leichtfertigkeit in keiner Hinsicht ausreichen kann ${ }^{156}$. Dies bewirkt eine wichtige Begrenzung der Haftung, $\mathrm{da}-$ anders als bei $\$ 826$ BGB -, das Korrektiv der Sittenwidrigkeit in $\$ 117$ Abs. 1 AktG nicht verlangt und auch durch die geforderte Nutzung einer Einflussnahmemöglichkeit insofern nicht hinreichend aufgewogen wird. Nur Täter, die sowohl im Bewusstsein ihrer Machtstellung als auch unter billigender Inkaufnahme des Schadenseintritts handeln, sollen $\$ 117$ Abs. 1 AktG unterliegen. Nicht zu verlangen ist demgegenüber auch ein schuldhaftes Handeln des Beeinflussten ${ }^{157}$, da andernfalls der Täter durch besonders geschickte, manipulative Weise einer Haftung entgehen könnte, wenn er sich im Übrigen - etwa mangels rechtswidriger Bereicherungsabsicht - nicht im Anwendungsbereich des $\$ 263$ Abs. 1 StGB bewegt.

152 Vgl. BGH WM 1981, 440, 442; OLG Koblenz GmbHR 1991, 416 f. Anderes kann auch nicht unter dem Regime der Business Judgement Rule gelten, vgl. BGHZ 135, 244, 253 und nunmehr $\$ 93$ Abs. 1 Satz 2 AktG, da auch mit dieser nicht die Aussage einhergeht, dass eine täuschungsbedingt herbeigeführte aber im Übrigen überprüfte objektiv pflichtwidrige Entscheidung irrtumsbedingt pflichtgemäß wird. Bereits das Zulassen des externen Einflusses muss insoweit Zweifel an der Anwendbarkeit von $₫ 93$ Abs. 1 Satz 2 AktG hervorrufen, Fleischer, in: Spindler/Stilz, AktG, 3. Aufl., 2015, $\mathbb{9} 93$ Rdn. 72 m. w. N., kritisch aber KrIEgER/SAILER, in: K. Schmidt/Lutter, AktG, 3. Aufl., 2015; $\$ 93$ Rdn. 15. Vgl. zur entsprechenden Diskussion einer Übertragbarkeit der BJR auf den Externen SpINDler, aaO (Fn. 49), \$117 AktG Rdn. 34 f.

153 Mit zutreffender Differenzierung nach Fehleinschätzungen und sonstigen Pflichtverletzungen Kort, aaO (Fn. 49), \$117 AktG Rdn. $201 \mathrm{ff}$.

154 Vgl. KorT, aaO (Fn. 49), \$117 AktG Rdn. 156 ff; ein wenig näher an das Erfordernis des dolus directus rückend Spindler, aaO (Fn. 49), $\$ 117$ AktG Rdn. 40, allerdings unter Bezugnahme von KorT, aaO, so dass davon auszugehen ist, dass auch SPINDLER dolus eventualis genügen lassen will und sich ausschließlich auf die kognitiven Elemente bezieht.

155 Vgl. zu den anerkannten Konstellationen Wagner, aaO (Fn. 7), \826 BGB Rdn. 26 ff, $29 \mathrm{ff} \mathrm{m.w.} \mathrm{N.}$

156 Vgl. Hueck/Windbichler, Gesellschaftsrecht, 23. Aufl., 2013, $\$ 23$ Rdn. 28.

157 Allg. M. vgl. nur Kort, aaO (Fn. 49), \117 AktG Rdn. 160. 
2. Gründe gegen eine Analogie zu $₫ 117$ Abs. 1 Akt $G$

a) Keine planwidrige Regelungslücke?

a) Die Verweisungsvorschrift des $\int 52 \mathrm{GmbHG}$

Auf der Grundlage der Haftungsstruktur des $₫ 117$ Abs. 1 AktG könnte zunächst mit Verweis auf $\$ 52 \mathrm{GmbHG}$ gegen das Vorliegen einer planwidrigen Regelungslücke argumentiert werden. In $\$ 52 \mathrm{GmbHG}$ werden diejenigen Vorschriften des AktG in Bezug genommen, die bei Bildung eines Aufsichtsrats in der $\mathrm{GmbH}$ (sei dies freiwillig oder auf Basis der Mitbestimmungsgesetze zwingend ${ }^{158}$ ) zur Anwendung kommen. In Bezug auf die Haftung ist dort $\$ 116$ AktG, nicht jedoch $\$ 117$ AktG genannt (Gleiches gilt für $\ 1$ Abs. $1 \mathrm{Nr} .3$ DrittelbtG). Insofern sei auch berücksichtigt, dass eine Aufnahme der Vorschrift in $₫ 52 \mathrm{GmbHG}$ durchaus nahegelegen hätte, da jedenfalls $₫ 117 \mathrm{Abs.} 2$ AktG keinen Jedermannparagraphen bildet und auf Aufsichtsratsmitglieder anwendbar ist ${ }^{159}$. Dieses formale Argument erscheint jedoch bei näherer Betrachtung schwach.

Zunächst weisen die $\$ \$ 93,116$ AktG, 52 GmbHG eine umfangreiche Haftung für Vorstand und Aufsichtsrat gegenüber der Gesellschaft wegen Pflichtverletzungen auf ${ }^{160}$. $\$ 117 \mathrm{AktG}$ hat daneben kaum praktische Bedeutung, soweit es nicht darum geht, dass Aktionäre über $\$ 117$ Abs. 1 Satz 2 AktG Schäden liquidieren wollen, die über bloße Reflexschäden hinausgehen ${ }^{161}$. Daraus ergibt sich, dass für den Gesetzgeber keine gesellschaftsrechtliche Notwendigkeit bestand, $\mathbb{} 117$ AktG ausdrücklich in $\$ 52 \mathrm{GmbHG}$ aufzunehmen. Da $\$ 117$ Abs. 1 Satz 1 AktG als allgemein deliktische Vorschrift zu werten ist, musste diese Haftung in $\$ 52 \mathrm{GmbHG}$ nicht berücksichtigt werden und gilt gleichwohl ebenso wie die $\$ \int 823 \mathrm{ff}$ BGB als deliktsrechtliche Jedermannhaftung. Allenfalls eine fehlende Aufnahme von $\$ 117$ Abs. 2 AktG könnte wegen des dort angesiedelten speziellen Bezugs auf die Aufsichtsratsstellung berücksichtigungsfähig sein, jedoch ergibt sich hieraus kein Argument gegen eine erweiterte Anwendung von $\$ 117 \mathrm{Abs} .1 \mathrm{AktG}$, der besagten personellen Bezugspunkt gerade nicht aufweist und somit eigenständig zu betrachten ist. $\$ 117$ Abs. 2 und $\$ 117$ Abs. 3 AktG hätten auch unproblematisch als $\$ \$ 117$ a, b AktG systematisiert werden können.

158 Gemäß $\$ 1$ Abs. 1 Nr.3 DrittelBG ab 501 Arbeitnehmer zwingend, da eine Drittelbesetzung mit Arbeitnehmervertretern im Aufsichtsrat vorgeschrieben ist und diese nur bei Existenz eines Aufsichtsrats ermöglicht wird.

159 Näher Kort, aaO (Fn. 49), $\$ 117$ AktG Rdn. 191 ff.

160 Vgl. Spindler, Münchener Komm. z. GmbHG, 2012, $\$ 52$ Rdn. 553 ff m. w. N.

161 Vgl. Kort, aaO (Fn. 49), \$117 AktG Rdn. 189 f. 
Weiterhin ist $\ 52 \mathrm{GmbHG}$ nicht als abschließende Aufzählung von inkludierten Vorschriften, sondern vielmehr als Regelungsvorschlag für den Fall anzusehen, dass die Gesellschaft keine spezifische Gestaltung durch Satzungsgebung kennt ${ }^{162}$. Wohl als zwingend dürfte die grundsätzliche Haftung des Aufsichtsrates gegenüber der Gesellschaft nach $\$ \mathbb{\$} 116,93$ AktG anzusehen sein, soweit auf diese Drittgläubiger im Wege der Pfändung und Überweisung oder der Insolvenzverwalter zugreifen wollen. Aber selbst in diesem Bereich werden Haftungserleichterungen etwa in Bezug auf den Sorgfaltsmaßstab diskutiert $^{163}$. Zuzustimmen ist dabei der Ansicht, zwischen fakultativem und obligatorischem Aufsichtsrat zu unterscheiden ${ }^{164}$.

Schließlich kann es kaum überzeugen, den Verweis des $\mathrm{GmbH}$-Rechts für die rechtliche Handhabung des Aufsichtsrates als Maßstab in der Analogiedebatte um $\$ 117$ Abs. 1 AktG heranzuziehen. Der Regelungs- und präventive Schutzgehalt des $\$ 117$ Abs. 1 AktG zielt auf die Abwehr äußerer Einflussnahme ${ }^{165}$, die sodann zu Fehlentscheidungen und anderen Pflichtverletzungen der Verwaltungsebene der Gesellschaft führen. Dieser „äußere“ Einfluss mag zwar durchaus auch von einem Aufsichtsratsmitglied stammen können (was denselben gleichsam als „inneren“ Einfluss erscheinen ließe) ${ }^{166}$, jedoch bewegt sich das Aufsichtsratsmitglied sodann einerseits immer zugleich im Regelungsbereich der $\mathbb{\int} 116,93 \mathrm{AktG}, 52 \mathrm{GmbHG}$ und andererseits ist die in die Pflicht genommene Verwaltungsebene, zu der auch der Aufsichtsrat gehört, gerade nicht der klassische Adressatenkreis, für den die zusätzliche Haftungsandrohung nach $\$ 117$ Abs. 1 AktG (und für Aufsichtsratsmitglieder nach Abs. 2!) geboten ist ${ }^{167}$.

\section{bb) Haftung für Feblinformationen}

In den unter II. erörterten Haftungsmechanismen wurde aufgezeigt, dass der rechtsgeschäftliche und deliktische Schutz gegenüber fehlerhaften oder pflichtwidrig unterschlagenen Informationen in erheblichem Maße aus-

162 Vgl. Altmeppen, in: Roth/Altmeppen, GmbHG, 8. Aufl., 2015, 552 Rdn. 1.

163 Vgl. OLG Brandenburg, ZIP 2009, $866 \mathrm{f} \mathrm{m.} \mathrm{Anm.} \mathrm{Schodder,} \mathrm{EWiR} \mathrm{2009,} 479$ f; Altmeppen, ZIP 2010, 1973, 1978. A. A. für den zwingenden Aufsichtsrat mit starken Argumenten SpIndler, aaO (Fn. 160), $\$ 52$ GmbHG Rdn. 555 ff.

164 Vgl. Spindler, aaO (Fn. 160), $\$ 52$ GmbHG Rdn. 555 ff.

165 Zutreffend kategorisiert von KorT, aaO (Fn. 49), $\$ 117$ AktG Rdn. 19.

166 Hierauf basiert auch die missverständliche Aussage des BGH, $\mathbb{1 1 7}$ AktG habe auch aus der gesellschaftsrechtlichen Treuepflicht heraus konzipiert werden können, vgl. BGHZ 129, 136, 160 = NJW 1995, 1739, 1745. Zutreffend hält Spindler, aaO (Fn. 49), $\$ 117$ AktG Rdn. 4 entgegen, dass bereits die Entstehungsgeschichte beweist, dass der heutige Jedermannparagraph gerade nicht aus der Treuepflicht hatte konzipiert werden können, da dieses Konzept nicht alle Fälle der Einflussnahme zum gebotenen Schutz der Gesellschaft zu erfassen vermag.

167 Vgl. Spindler, aaO (Fn. 49), \$117 AktG Rdn. 3, 16 ff. 
geprägt ist. Schärfere oder andersartige Haftung respektive eine Haftung für andere Konstellationen erscheinen nach hier vertretener Ansicht nicht geboten. Soweit die rechtswidrige Einflussnahme Dritter sich also des Mittels der Falschinformation bedient, träte $\mathbb{1 1 7}$ Abs. $1 \mathrm{AktG}$ in analoger Anwendung allenfalls neben die gegebenen Haftungsinstrumente, jedoch bestünde kein Bedürfnis der Ergänzung. Daraus ist allerdings nicht zu folgern, dass generell für den Bereich der rechtswidrigen Einflussnahme von Verwaltungsmitgliedern eine planwidrige Regelungslücke abzulehnen wäre. Es handelt sich lediglich um eine zufällige Überschneidung von Regelungsbereichen, die einander jedoch nicht bedingen oder gar gegenseitig ausschließen. Ein Vergleich zeigt, dass die Haftung für Fehlinformationen ein ganz bestimmtes Schädigerverhalten in jedem Fall mit einer Haftungsvorschrift abwehren oder Restitution bei kausal entstandenem Schaden bieten will. Dies wird über verschiedene Vehikel aus Vertrag und Delikt erreicht ${ }^{168}$. Fehlinformationen können gleichermaßen Tatbestände nach dem UWG auslösen ${ }^{169}$ oder auf Umwegen eine

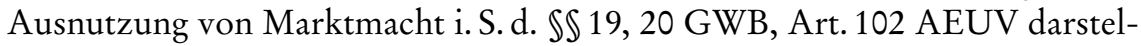
len ${ }^{170}$. Eine derart spezifische Abwehr eines bestimmten Schädigungsmittels bietet keinen verlässlichen systemkongruenten Ansatz, um hieraus Rückschlüsse für oder gegen andersartige Haftungsgründe zu finden, die lediglich rein zufällig auch im Fall der Fehlinformation ausgelöst werden können.

\section{cc) Abschließendes System durch die $\iint 823$ Abs. 1, 2 und 826 BGB?}

Unter II. wurden die allgemeinen deliktischen Haftungsmechanismen ausführlich erörtert. Die Analyse förderte in Bezug auf die Haftung für externe Beeinflussung der Verwaltungsebene der $\mathrm{GmbH}$ einen unsteten „Flickenteppich“ zu Tage. Bereits aus diesem Grund erscheint es unstatthaft, den Rückschluss ziehen zu wollen, dass das allgemeine deliktische System für den Fall externer Einflüsse auf die Gesellschaftsleitung als abschließend zu werten sei, auch wenn zuzugeben ist, dass fragmentarischer Haftung freiheitssichernde Wirkung innewohnt ${ }^{171}$. Entscheidendes Gegenargument wider einer solchen These ist letztlich aber die Existenz von $\$ 117$ Abs. 1 AktG selbst. Es kann nicht argumentiert werden, dass der Gesetzgeber für die AG eine Grundsatz-

168 S. o. III.1. und III.6.

169 Insbesondere kommen Formen unzulässiger Beeinträchtigung der Entscheidungsfreiheit nach den $\$ \mathbb{S} 3,4$ Nr. 1 UWG, vgl. HasselblatT, in: Gloy/Loschelder/Erdmann, Hdb. des Wettbewerbsrechts, $\$ 48$ Rdn. $47 \mathrm{ff}, 78 \mathrm{ff}$, und unzulässiger Betriebsstörung als Form der Mitbewerberbehinderung nach den $\$ \$ 3,4$ Nr. 10 UWG in Betracht, vgl. Hasselblatt, aaO, $\$ 57$ Rdn. 154 ff jeweils m. w. N.

170 Dies kommt insbesondere in Form unbilliger Behinderung nach $\$ 19$ Abs. 2 Nr. 1 GWB in Betracht, sofern die Marktmacht gerade auch zur Einflussnahmemöglichkeit durch Information führt, vgl. die instruktiven Ausführungen der Beschlusskammer der BNetzAgentur v. 23. 09. 2010, Az. BK 2b 09/010, insbesondere S. $18 \mathrm{ff}$.

171 So zutreffend Weller, FS Hoffmann-Becking, 2013, S. $1341 \mathrm{ff}$. 
entscheidung in Bezug auf die Haftung für externe Einflussnahme statuierte, was als allgemeiner Deliktsschutz ausgestaltet wurde, und zugleich die Aussage hatte verfolgen oder auch nur akzeptieren wollen, dass die $\$ \int 823 \mathrm{ff}$ BGB im Übrigen eben jenen Regelungsbereich umfassend abdecken sollen. Dies stellte konkurrenzrechtlich einen diametralen Widerspruch dar, der weder den $\int \mathbb{8} 823 \mathrm{ff}$ BGB noch $\$ 117 \mathrm{Abs} .1 \mathrm{AktG}$ entnommen werden kann.

\section{dd) Einwand der Ferne von Leitung und Rechtsinhaberschaft (Servatius und Schall)}

Servatius ${ }^{172}$ und Schall ${ }^{173}$ wollen die Analogiefähigkeit des $\ 117$ Abs. 1 AktG grundsätzlich auf jene Fälle beschränken, in denen in der $\mathrm{GmbH}$ ebenso wie in der AG eine erhebliche Ferne zwischen Geschäftsleitung und Gesellschafterebene besteht. Eine Analogie soll also nur in Betracht kommen, wenn ein Aufsichtsrat eingerichtet ist, da nur in diesem Fall der Schutzgedanke gegen den einflussnehmenden Großaktionär auf die $\mathrm{GmbH}$ übertragen werden könne. $\$ 117$ Abs. 1 AktG stehe in systematisch und teleologisch untrennbarem Konnex zum Gewaltenteilungsprinzip der AG, $\mathbb{S} 576,119$ Abs. 2 AktG ${ }^{174}$.

Diese Einwände greifen angesichts der hier geführten Debatte nicht durch. Die so eingeschränkte Betrachtung vermag bereits nicht zu erklären, weshalb sich der Gesetzgeber vom Leitbild der Haftung des Großaktionärs abwandte und sogar einen Jedermannparagraphen für erforderlich hielt. Dementsprechend ist auch das Zusatzargument von Schall, die GmbH-Gesellschafter dürften unter Wahrung von Minderheitenschutz und bei Vermeidung von Gläubigerschädigung in der Insolvenz ihre eigene Gesellschaft beliebig schädigen ${ }^{175}$, nicht durchschlagend. $\$ 117$ Abs. 1 AktG böte in seiner analogen Anwendung allenfalls selbst ein weiteres Korrektiv für treugemäßen Minderheitenschutz. Zudem ist die Einflussnahme auf alle wesentlichen Geschäftsleiter ausgedehnt worden, wenn sogar Prokuristen und Handlungsbevollmächtigte taugliche Einflussnahmesubjekte sind. Ein spezifischer Zusammenhang zur Machtausübung des Großaktionärs ist darin nicht zu erkennen. Entscheidend ist jedoch das Argument, dass die obige Untersuchung relevante Haftungslücken zeigen konnte, die in der AG nicht existieren, deren tatsächliche Konstellationen und schädigende Konsequenzen aber gleichsam in der $\mathrm{GmbH}$ nicht akzeptiert werden dürfen ${ }^{176}$.

172 Servatius, Gläubigereinfluss durch Covenants, 2008, S. $138 \mathrm{f}$.

173 Schall, aaO (Fn. 118), $\$ 117$ AktG Rdn. 1, 34.

174 Servatius, aaO (Fn. 172), S. 138 f; Schall, aaO (Fn. 118), $\$ 117$ AktG Rdn. 1, 34.

175 Schall, aaO (Fn. 118), $\$ 117$ AktG Rdn. 1, 34.

176 A. A. Servatius, aaO (Fn. 172), S. $138 \mathrm{f}$. 
b) Kein vergleichbarer Regelungssachverbalt?

\section{aa) Feblende Übertragbarkeit der Beschränkung von Verzicht und Vergleich?}

Fehlende Vergleichbarkeit könnte allenfalls im Bereich der Ausschlusstatbestände nach den $\int \mathbb{S} 117$ Abs. 4, 93 Abs. 4 Satz 3 AktG erwogen werden. Dieses Argument wird vermeintlich durch einen Blick in die $\mathbb{S} 9 \mathrm{~b}, 43$ Abs. 3 Satz 2 GmbHG gestützt, die erleichterte Mechanismen für Verzicht und Vergleich über Ansprüche gegen Verwaltungsmitglieder vorsehen.

Soweit es für unerlässlich gehalten wird, $\$ 117$ Abs. 4 AktG bei der analogen Anwendung des $\$ 117$ Abs. $1 \mathrm{AktG}$ ebenfalls heranzuziehen, ist dieser Ansatz aber jedenfalls bereits durch die gesetzgeberische Entscheidung in $\$ 52$

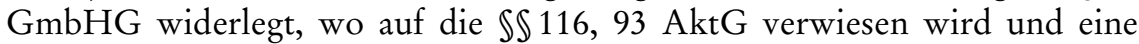
Übertragung des $₫ 93$ Abs. 4 Satz $3 \mathrm{AktG}$ - jedenfalls für den obligatorischen Aufsichtsrat der $\mathrm{GmbH}$ - überwiegend als akzeptabel anzusehen ist ${ }^{177}$. Freilich wird dabei nicht übersehen, dass es in der Sache durchaus Probleme bereitet, die Entscheidung der Hauptversammlung und das Argument des Minderheitenschutzes in $\$ 93$ Abs. 4 AktG auf die $\mathrm{GmbH}$ zu übertragen ${ }^{178}$. Die $\mathrm{GmbH}$ weist mit ihrer gesellschafterbezogenen Führungsstruktur ( $\$ 37$ Abs. $1 \mathrm{GmbHG}$ ) a priori starke Instrumente zur Intervention seitens der Inhaberebene auf und kennt eigene Formen des Minderheitenschutzes (bspw. $\$ \mathbb{5} 50,51$ a GmbHG). Gleichwohl ergibt sich hieraus kein Argument, $\mathbb{9} 93$ Abs. 4 Satz 3 AktG für unanwendbar zu erachten, da die Abwägung schutzwürdiger Belange mit der eingebauten Verzichts- und Vergleichssperre gesetzgeberisch vorgenommen und nicht als unbillig abgetan werden kann. Selbst wenn man dies anders sähe, ergäbe sich hieraus das Gebot einer teleologischen Reduktion bei der Übertragung auf die $\mathrm{GmbH}^{179}$, sicherlich aber kein Argument gegen die Anwendung des $\$ 117 \mathrm{AktG}$ schlechthin.

\section{bb) Wertungen des Konzernrechts}

Schließlich ergeben sich auch aus den internen konkurrenzrechtlichen Erwägungen des Aktiengesetzes keine Argumente gegen eine vergleichbare Interessenlage. Wenngleich $\$ 117$ Abs. 1 AktG in der Haftungsstruktur der AG wegen der - nicht auf die $\mathrm{GmbH}$ übertragenen ${ }^{180}$ - Konzernhaftungstatbestände

177 Vgl. Zöllner/Noack, in: Baumbach/Hueck, GmbHG, 20. Aufl., 2013, \52 Rdn. 70, 210; Lutter/Hommelhoff, GmbHG, 18. Aufl., 2012, \52 Rdn.63; a. A. Scholz/ Schneider, aaO (Fn. 42), \52 GmbHG Rdn. 524.

178 Vgl. Spindler, aaO (Fn. 160), $\$ 52$ GmbHG Rdn. 618 f.

179 Diese vornehmend SpIndler, aaO (Fn. 160), $\$ 52$ GmbHG Rdn. 618. A. A. allerdings ohne Diskussion Heermann, aaO (Fn. 120), $\$ 52$ GmbHG Rdn. 64.

180 So jedenfalls für $\$ 311$ AktG, vgl. Liebscher, Münchener Komm. z. GmbHG, 2. Aufl., 2015, Anhang $\ 13$ Rdn. 317 ff; Wicke, GmbHG, 2. Aufl., 2011, Anhang $\ 13$ Rdn. 11. 


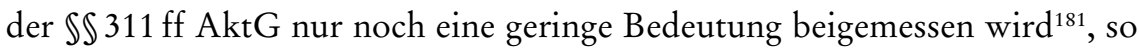
kann dies gleichwohl für die vorliegende Erörterung kein Gegenargument bilden. $\$ 117$ Abs. 1 AktG mag in seiner Entwicklungsgeschichte manches $\mathrm{Mal}$ als Form einer Konzernhaftung verstanden worden sein, jedoch dürfte diese Sichtweise spätestens mit der Zustimmung verdienenden Verselbstständigung des Konzernrechts obsolet sein. Auch weist die Gesetzessystematik darauf hin, dass $\$ 117$ Abs. 1 AktG eigenständig zu verstehen ist, ist dieser doch ohne unmittelbar systematische Anknüpfung direkt hinter den Vorschriften zum Aufsichtsrat platziert. Die Schwierigkeit des Gesetzgebers, eine systematisch sinnvolle Stelle zu finden, erklärt sich eben daraus, dass die Vorschrift einem allgemeinen Teil des Gesellschaftsrechts zuzuordnen ist und somit ein Haftungsprinzip beinhaltet, welches weder AG-spezifisch noch konzernrechtlich eingestuft werden darf ${ }^{182}$.

\section{Positivkriterien der Analogie}

\section{a) Gesetzgebungsmaterialien}

Aus den Materialien lässt sich für die vorliegende Diskussion nur wenig entnehmen. An keiner Stelle der Entwürfe zur Schaffung des $\$ 101 \mathrm{AktG} 1937^{183}$ oder des $\ 117$ AktG $1965^{184}$ ist positiv verzeichnet, dass dieser Haftungstatbestand Ausdruck eines allgemeinen Prinzips sein könnte, oder negativ festgestellt, dass es sich um eine speziell und ausschließlich für das Aktienrecht geltende Haftungsstruktur handeln soll. Allerdings wird die Annahme bestätigt, dass bereits $\$ 101 \mathrm{AktG} 1937$ als Vorläufer des heutigen $\$ 117$ AktG keine spezifisch gesellschaftsrechtliche Haftung, sondern einen besonderen Deliktstatbestand i. S. d. $\iint 823 \mathrm{ff}$ BGB darstellte ${ }^{185}$. Dies beweist die Abkehr von der zunächst vor 1937 avisierten reinen Aktionärshaftung mit dem Erlass eines Jedermannparagraphen ${ }^{186}$.

181 Vgl. Spindler, aaO (Fn. 49), $\$ 117$ AktG Rdn. 3.

182 Näher zur allgemein deliktischen Einordnung sogleich unter III.3.

183 RGBl. 1937 I Nr. 15, S. 107-165, ber. S. 588, 1140.

184 BGBl. I 1965, S. 1089.

185 Hierzu sei auf die umfassende Darstellung bei Voigt, aaO (Fn. 118), S. 5 ff, 19 ff verwiesen, der schwer nachvollziehbar gleichwohl an späterer Stelle zu dem Ergebnis gelangt, dass gerade $\$ 101$ AktG 1937 nicht als Deliktstatbestand aufzufassen sei, Voigt, aaO, S. $207 \mathrm{ff}, 219 \mathrm{f}$.

186 Hierzu Dietrich, JW 1937, 649, 654; Voigt, aaO (Fn. 118), S. 20 m. w. N. 


\section{b) Gesetzgebungsgeschichte und die Unzulänglichkeit des Sittenwidrigkeitsverdikts}

Was jedoch die Geschichte hin zur Entstehung des $\$ 101$ AktG 1937 deutlich zeigt, ist der Umstand, dass für Fälle externer Ausnutzung besonderer Einflussnahmemöglichkeiten die allgemeinen Deliktstatbestände nach Ansicht des Aktienreformgesetzgebers nicht genügten. Ausgehend von dem Problem sittenwidriger Stimmrechtsausübung durch Großaktionäre ${ }^{187}$ entbrannte eine Debatte, wie man unzulässigen Einflussnahmen Herr werden könnte, wobei man sich jedoch gerade vom Leitbild der Haftung des Großaktionärs gelöst hat ${ }^{188}$. Der bis dahin präferierte Ansatz über das Sittenwidrigkeitsverdikt wurde schnell als unzureichend und unpassend entlarvt ${ }^{189}$. Dem ist auf Basis obiger Erörterungen zu $\ 826$ BGB auch heute nach wie vor zuzustimmen. Die Generalklausel des Deliktsrechts für den Ersatz primärer Vermögensschäden ist weder für eine allgemeine Ausdehnung im wirtschaftlichen Leistungswettbewerb konstruiert ${ }^{190}$, noch darf sie dazu führen, dem Richter die Schaffung umfassender Ordnungsvorgaben in diesem Bereich aufzubürden $^{191}$. Es ist nicht Sache der Gerichte, über das Deliktsrecht eine eigenständig denkende Wettbewerbsordnung neben jener zu erschaffen, die der Gesetzgeber primär mit BGB, GWB, UWG sowie zahlreichen Nebenund Spezialgesetzen geschaffen hat. Zu Recht wird $\$ 826$ BGB daher als Auffangtatbestand ${ }^{192}$ und nicht als allgemeine Entwicklungsklausel der Wettbewerbsordnung aufgefasst. Die $\$ 826$ BGB zuerkannte Entwicklungsfunk-

187 So hatte das Reichsgericht begonnen, Fallkonstellationen der Einflussnahme außerhalb des gesellschaftsrechtlichen Kompetenzgefüges mit einer Haftung über $\$ 826$ BGB zu belegen, vgl. RGZ 107, $72 \mathrm{ff}$ und $202 \mathrm{ff} ; 108,41 \mathrm{ff}$ und $322 \mathrm{ff} ; 111,26 \mathrm{ff} ; 132$, $149 \mathrm{ff} ; 146,385 \mathrm{ff}$.

188 Eingehend dargestellt bei Voigt, aaO (Fn. 118), S. $5 \mathrm{ff}$.

189 Vgl. Voigt, aaO (Fn. 118), S. 7 ff m.N. zur Aktienrechtskommission des 34. DJT und zu den Erörterungen des Enquête-Ausschusses seit 1926.

190 Vgl. Wagner, aaO (Fn. 7), $\$ 826$ BGB Rdn. 4 ff. Zum Hintergrund, treffend mit dem Begriff des fehlenden „sozialen Schadens“ besetzt, WAGNER, aaO, Rdn. 13.

191 Mit entsprechend zweifelnder Zurückhaltung formuliert auch WAGNER, aaO (Fn. 7), \826 BGB Rdn. 18: „Dabei kommen die Gerichte nicht umhin, auch rechtspolitische Erwägungen über die sozialen Folgen der Qualifikation als sittenwidrig und über die daran angeschlossene Haftung anzustellen." Instruktiv hierzu Mertens, AcP 178 (1978), 227 ff. Zur Konsequenz der Schaffung von Präjudizien ausführlich OHLY, AcP 201 (2001), 1 ff. Für das Recht am Unternehmen mit entsprechenden Erwägungen Hager, aaO (Fn. 23), $\$ 823$ BGB Rdn. D 4.

192 Vgl. hierzu BachmanN, Steuerungsfunktion des Haftungsrechts im Gesellschafts- und Kapitalmarktrecht, 2007, S.93, 130 ff. S. a. OechsLer, aaO (Fn. 82), \826 BGB Rdn. 20. Deutlich wird dies insbesondere in der Konkurrenz zum Vertragsrecht, dessen Wertungen und damit verbundenen Güterzuweisungen es zu achten gilt, vgl. WAGNer, aaO (Fn. 7), \826 BGB Rdn. 15. 
tion $^{193}$ bezieht sich somit auf im Wesentlichen nicht regulierte und im täglichen Leben inakzeptable Szenarien, bei welchen dem im Einzelfall erkennenden Gericht bewusst ein vom Gesetzgeber nicht abschließender Spielraum für Haftungszuweisung belassen wird ${ }^{194}$.

Ein derartiges Ausfüllungsmoment mag das RG gerade für rechtswidrigen Einfluss erkannt haben ${ }^{195}$, was nachvollziehbar ist. Schon das RG hatte aber offenkundig Schwierigkeiten, eine generelle, belastbare und damit rechtssichere Linie zu finden, die der Rezeptions-, Transformations- und Delegationsfunktion ${ }^{196}$ der deliktischen Generalklausel für dauerhaft anerkennungsfähige Entscheidungen einen Haltepunkt bieten könnte. Auch die in $\$ 826$ BGB angelegte methodische Anweisung zur Interessenabwägung ${ }^{197}$ im Einzelfall beweist, dass ein geschlossener Haftungstatbestand, der die Fälle externer Einflussnahme beschränkt, sowohl passender als auch praktikabler ist. Daraus folgt im Ergebnis, dass ein bereits vom RG erkanntes Bedürfnis für die Schaffung einer Haftungsklausel zum Schutz vor unzulässigen Einflussnahmen besteht und dieses Bedürfnis offensichtlich gerade nicht nur an die Aktiengesellschaft zu binden ist. Interessanterweise wurde dies bereits in den Diskussionen um die Jahre 1930 und 1931 von Kißkalt fast beiläufig angesprochen ${ }^{198}$, jedoch ob der Fixierung auf das Aktienrecht nicht zum Gegenstand einer weiterführenden Debatte erhoben.

c) Die Ausgestaltung des $₫ 117$ Abs. 1 AktG und der Schutzzweck der Norm

\section{aa) Personell}

Personell soll die Haftung dem Wortlaut entsprechend nur der „Gesellschaft“, was aufgrund der Systematik im AktG ausschließlich die AG meinen kann, und nach $\$ 117$ Abs. 1 Satz 2 AktG außerhalb bloßer Reflexschäden auch dem

193 Vgl. etwa OTt, FS Raiser, 1974, S. 403 ff; BAUdenbacher, GRUR 1981, 19; SACK, WRP $1974,247 \mathrm{ff}, 251 \mathrm{ff}$.

194 Dementsprechend wird die Subsidiarität bei der positiven Bestimmung des Sittenwidrigkeitsmerkmals betont, vgl. Bachmann, aaO (Fn. 192); Wagner, aaO (Fn. 7), $₫ 826$ BGB Rdn. 18.

195 Vgl. RGZ 107, $72 \mathrm{ff}$.

196 Vgl. Teubner, Generalklauseln als sozionormative Modelle, in: Generalklauseln als Gegenstand der Sozialwissenschaften, 1978, S. 13 ff.

197 Vgl. Förster, AcP 209 (2009), 398, 409 f. Zum geschichtlichen Hintergrund KatZeNMEIER, AcP 203 (2003), 79 ff. Mit dem Versuch einer grundsätzlichen Qualifikation des Normzwecks Deutsch, NJW 1963, 385, $389 \mathrm{ff}$.

198 Vgl. Kisskalt, in: Schubert et al., Akademie für Deutsches Recht, Bd. I, 1986, S. 484. Daneben weist K. SснміDT, ZHR 171 (2007), 2, 5 in anderem Zusammenhang darauf hin, dass das GmbH-Recht über die Zeit hinweg kaum Änderungen erfahren hat und nur unmaßgeblich an der Entwicklung teilnahm. 
einzelnen geschädigten Aktionär zustehen. Für diese privilegierte Stellung der $A G$ und der Aktionäre gegenüber der $\mathrm{GmbH}$ ist kein tragfähiger Grund ersichtlich. Weder kann behauptet werden, dass die AG das grundsätzlich schutzwürdigere Angriffsziel solcher Attacken im Vergleich zur GmbH wäre, noch erscheint das Argument belastbar, GmbHs könnten sich effektiver verteidigen, da die Gesellschafterebene unmittelbar via $\$ 37$ Abs. $1 \mathrm{GmbHG}$ über die Verwaltungsebene herrsche. Es kann nicht ernsthaft bezweifelt werden, dass Geschäftsführung, Aufsichtsrat und berufene handelsrechtliche Vertreter der $\mathrm{GmbH}$ ebenso einer rechtswidrigen Beeinflussung unterliegen können, wie dies bei der AG der Fall sein kann. Auch drohen der GmbH dieselben Arten von Schäden und derselbe Schadensumfang. Ob der nicht zu leugnenden wirtschaftlichen Bedeutung der $\mathrm{GmbH}$-geführten Unternehmen in Deutschland will wohl auch niemand behaupten, dass die drohenden Auswirkungen auf alle denkbaren Stakeholderinteressen gegenüber der Situation der in Deutschland ansässigen AGs zu vernachlässigen wäre.

\section{bb) Sachlich}

aaa) Wird der Tatbestand des $\$ 117$ Abs. 1 AktG seinem Schutzzweck nach näher betrachtet, so fällt insbesondere die Einbeziehung der Beeinflussung von Prokuristen und Handlungsbevollmächtigten auf. Hieraus ist zwingend $\mathrm{zu}$ folgern, dass mit $\$ 117$ Abs. 1 AktG jedenfalls nicht auf rechtliche oder tatsächliche Unterschiede zwischen Vorstand und Aufsichtsrat in der AG einerseits und Geschäftsführung und Aufsichtsrat in der $\mathrm{GmbH}$ andererseits Rücksicht genommen wurde. Prokuristen und Handlungsbevollmächtigte können in der $\mathrm{GmbH}$ gleichfalls eingesetzt werden und unterliegen denselben rechtlichen Bestimmungen und tatsächlichen Gegebenheiten wie bei der Beauftragung durch die AG. Der besagte Schutzumfang zeigt vielmehr, dass es in $\$ 117$ Abs. 1 AktG um die Sicherung der Integrität all jener Entscheidungsträger geht, die rechtlich bindend agieren können und somit das Potential besitzen, der Gesellschaft schweren Schaden von innen heraus zuzufügen ${ }^{199}$. Nur die Wahrung der Integrität dieser Entscheidungsträger bietet der Gesellschaft und ihren Anteilseignern die Möglichkeit, auf die ordnungsgemäße treuhänderische Verwaltung ihrer Interessen Vertrauen zu können und ihr Vermögen gesichert zu wissen ${ }^{200}$.

199 Vgl. Spindler, aaO (Fn. 49), $\$ 117$ AktG Rdn. 1 f m. w. N. SPIndler, wie auch einige andere beziehen allerdings zusätzlich die Autonomie der Willensbildung des jeweiligen Verwaltungsorgans mit ein, was sich weder trennscharf definieren lässt noch aus Geschichte oder objektiver Teleologie der Norm entnommen werden kann. Für diese überschießende Normzweckerklärung besteht weder Not noch Bedarf; sie ist abzulehnen.

200 Anderes kann auch vom Schutzumfang nicht getragen werden, da die Verwaltungsmitglieder in Bezug auf die Gesellschaft wegen ihrer treuhänderischen Stellung gerade 
$b b b$ ) Unmittelbar an die vorangegangenen Ausführungen (aaa) schließen sich folgende Überlegungen an: Für einen arbeitsteiligen Wirtschaftsverkehr ist die angesprochene Verlässlichkeit der Verwaltungsorgane unabdingbar. Will das deutsche Gesellschaftsrecht eine wettbewerbsfähige Ordnungspolitik im Leistungsstreit der Rechtsordnungen anbieten ${ }^{201}$, muss berücksichtigt werden, dass die auf Dauer angelegte Verbindung von sächlichen und immateriellen Ressourcen mit einer spezifischen Zweckbindung nach dem deutschen numerus clausus der Gesellschaftsrechte ${ }^{202}$ immer in den Verbund einer gesellschaftsrechtlichen Struktur zwingt, sofern kein einzelkaufmännisches Unternehmen betrieben wird. Letzteres steht aber außerhalb der hier zu erörternden Konkurrenz, da dieses gerade nur sehr eingeschränkt eine verlässliche Arbeitsteilung erlaubt. Zudem kann der Schutz vor privater Haftung ausschließlich mittels bestimmter Gesellschaftsformen erreicht werden (im Wesentlichen durch Errichtung einer KG oder einer Kapitalgesellschaft). Somit ist der Schutz der Integrität des Verwaltungshandelns zugleich integraler Bestandteil einer guten gesellschaftsrechtlichen Ordnungspolitik und ein Schutz gegen Marktversagen, da sich der Markt an dieser Stelle gerade mangels hinreichender Mechanismen nicht selbst zu regulieren vermag. Insofern droht die kompensationslose Vernichtung von Ressourcen der Gesellschaft, so dass Prävention gegen das Verhalten schädigender, rechtswidriger Beeinflussung geboten ist. Dem stehen keine schutzwürdigen Marktinteressen der Einflussnehmer gegenüber, die in Bezug auf die Ressourcen der Gesellschaft weder aus Gesichtspunkten des Wettbewerbs noch aus Ansätzen sozialer Teilhabe Ansprüche herleiten könnten. Diese Erkenntnisse stimmen sodann zudem mit der höchstrichterlichen Ansicht überein und bilden gleichsam ihren Hintergrund, dass Verletzungen der gesellschaftsrechtlichen Treuepflicht schwerer wiegen sollen, als dies bei schlichten Verletzungen von Austauschverträgen der Fall ist ${ }^{203}$. Dieser Rechtsprechungsansatz ist aus ökonomischer Sicht insbesondere im Hinblick auf gesellschaftsrechtlich verbundenes Vermögen zutreffend, wenn es um die Schaffung sinnvoller Investitionsanreize für Gesellschafter geht. Größere unternehmerische Vorhaben können regelmäßig nicht ohne entsprechende Kapitalsammlung verwirklicht werden, so dass ein Marktversagen in diesem Bereich erhebliche negative Auswirkungen zeitigen kann. Schließlich können diese Ziele zwar auch ausschließlich durch Pflichtenbindung und Haftung des jeweils beeinflussten Geschäftsleiters verfolgt werden, jedoch kann der Vertrauensgewinn in die deutsche GmbH-

keine eigenen Interessen geltend machen dürfen, vgl. hierzu ausführlich KoRT, aaO (Fn. 49), $\$ 117$ AktG Rdn. 5 ff.

201 Zum Grundgedanken ausführlich Sandrock/WetZler, Deutsches Gesellschaftsrecht im Wettbewerb der Rechtsordnungen: Nach Centros, Überseering und Inspire Art, 2004.

202 Statt vieler Roth/Weller, Handels- und Gesellschaftsrecht, 8. Aufl., 2013, S. 65.

203 Vgl. BGHZ 12, $308 \mathrm{ff}=$ NJW 1954, $1159 \mathrm{ff}$. Zur der zu Grunde liegenden Fallgruppe des $₫ 826$ BGB s. o. III.6.c.cc. 
Rechtsordnung durch zusätzliche Inpflichtnahme des Einflussnehmers gestärkt werden, ohne das hierdurch schutzwürdige Belange oder Freiheiten zu beschneiden sind.

ccc) Als dritte Begründungslinie für die Notwendigkeit einer Übertragung von $\$ 117$ Abs. 1 AktG auf die GmbH im Bereich sachlich-inhaltlicher Erwägungen ist auf die bereits angesprochene Rechtsnatur der Vorschrift zurückzukommen. $\$ 117$ Abs. 1 AktG wird zutreffend als allgemeines Deliktsrecht eingeordnet und ist schon von diesem Standpunkt aus nicht an eine bestimmte Gesellschaftsform gebunden. Weder geht es darum, der AG speziell Schutz vor einem übermächtigen Großaktionär zu bieten, noch verlangt $\$ 117 \mathrm{Abs.} 1$ AktG die Verletzung einer gesellschaftsrechtlichen Treuepflicht oder basiert auch nur auf jener. Der Schädiger kann gerade auch vollständig außerhalb des gesellschaftsrechtlichen Geschehens stehen. Dies wird zusätzlich durch einen Vergleich der $\$ \mathbb{\$} 93,116$ mit $\$ 117$ Abs. 1 AktG verdeutlicht.

ddd) Schließlich bietet $\$ 117$ Abs. 1 AktG auch einen handhabbaren Haftungstatbestand, der Unternehmensschutz bei gesellschaftsrechtlich gebundenen Unternehmensressourcen vermittelt, ohne vorab auf die kaum rechtssicher vorzunehmende Abgrenzung angewiesen zu sein, ob besagter Schutz im Einzelfall gewährt werden darf. Dies zeigt sowohl den entscheidenden praktischen Vorteil als auch die dogmatisch bessere Verortung gegenüber $\$ 823$ Abs. 1 BGB, da mit $\$ 117$ Abs. 1 AktG eine rechtssichere Judikatur möglich ist, was vom Rahmenrecht des eingerichteten und ausgeübten Gewerbebetriebs kaum behauptet werden kann ${ }^{204}$. Auch bietet die Haftung nach $\$ 117$ Abs. 1 AktG mit den Erfordernissen des Vorsatzes und der nachzuweisenden Einflussnahme auf Basis einer entsprechenden Machtposition hinreichende Eingrenzungsmerkmale, die einer ausufernden Haftung und einer damit einhergehenden übermäßigen Beschränkung der allgemeinen Handlungsfreiheit entgegenwirken.

In der Gesamtschau ergibt sich mithin, dass eine Analogie zu $\mathbb{} 117$ Abs. 1 AktG im Fall rechtswidriger Einflussnahmen auf Verwaltungsmitglieder einer $\mathrm{GmbH}$ nicht nur zulässig, sondern geboten ist.

\section{Zusammenfassung in Thesenform}

1. Anders als die AG kennt das Recht der GmbH keinen gesetzlich explizit angeordneten haftungsrechtlichen Schutz gegen vorsätzliche Schädigungen durch externe Beeinflussung. Dies basiert jedoch nicht auf strukturellen Unterschieden, sondern erweist sich in einer eingehenden Analyse als planwidrige Regelungslücke. 
2. Die Haftungsmechanismen des bürgerlichen Rechts vermögen die Schutzlücke im Recht der $\mathrm{GmbH}$ nur partiell zu kompensieren. Dieser haftungsrechtliche „Flickenteppich“ lässt sich jedoch weder aus Unterschieden zur AG noch aus anderen Erwägungen heraus erklären. Es handelt sich um eine nicht überzeugende Diskriminierung von $\mathrm{GmbH}$ gegenüber AG-Sachverhalten bei ceteris paribus gleichgelagerten Interessen.

3. $\ 117$ Abs. 1 AktG weist einen allgemeinen Rechtsgedanken dahingehend auf, dass externe Einwirkungen auf Basis besonderer Einflussnahmemöglichkeiten kraft Rechtsgeschäfts oder faktischer Positionen nicht vorsätzlich genutzt werden dürfen, um der Gesellschaft Schaden zuzufügen. Dies hat der Gesetzgeber bei der Schaffung eines ordnungspolitischen Rahmens im Gesellschaftsrecht für die AG gebilligt und eine deliktische Haftung im Schädigungsfall angeordnet.

4. $\$ 117$ Abs. 1 AktG ist sowohl nach seinem Normzweck als auch in Bezug auf die personellen und sachlichen Voraussetzungen auf die $\mathrm{GmbH}$ übertragbar. Die Übertragung wird hier lediglich als Teilanalogie vorgeschlagen, so dass vorliegend keine abschließende Stellungnahme zum Gesamtkonzept des $₫ 117$ Abs. 2 - 7 AktG angeboten wurde, die einer eigenständigen wissenschaftlichen Erörterung vorbehalten bleibt.

5. $\$ 117$ Abs. 1 AktG kommt ohne das Sittenwidrigkeitsverdikt aus und bietet - verstanden als geschlossener Tatbestand - eine nachvollziehbare und damit klare Haftungsstruktur, die an gegebene Einflusspositionen anknüpft und einen schärferen Haftungsansatz als $\$ 826$ BGB bietet.

6. Darüber hinaus zeigt eine eingehende Analyse dogmatischer und wirtschaftspolitischer Hintergründe ein Bedürfnis für die Erweiterung dieses Haftungstatbestandes. $\$ 117$ Abs. 1 AktG ist daher auf die $\mathrm{GmbH}$ analog anzuwenden. 